\title{
Primordial helium abundance determination using sulphur as metallicity tracer
}

\author{
Vital Fernández ${ }^{1 \star}$, Elena Terlevich ${ }^{1}$, Angeles I. Díaz ${ }^{2,3,4}$, Roberto Terlevich ${ }^{1,5}$, \\ F. F. Rosales-Ortega ${ }^{1}$ \\ ${ }^{1}$ Instituto Nacional de Astrofísica, Óptica y Electrónica, Luis E. Erro 1, 72840 Tonantzintla, Puebla, Mexico \\ ${ }^{2}$ Departamento de Física Teórica, Universidad Autónoma de Madrid, E-28049 Madrid, Spain \\ ${ }^{3}$ Centro de Investigación Avanzada en Física Fundamental CIAFF-UAM \\ ${ }^{4}$ Astro-UAM, UAM, Unidad Asociada CSIC \\ ${ }^{5}$ Institute of Astronomy, University of Cambridge, Cambridge, UK
}

Accepted XXX. Received YYY; in original form ZZZ

\begin{abstract}
The primordial helium abundance $Y_{P}$ is calculated using sulphur as metallicity tracer in the classical methodology (with $Y_{P}$ as an extrapolation of $Y$ to zero metals). The calculated value, $Y_{P, S}=0.244 \pm 0.006$, is in good agreement with the estimate from the Planck experiment, as well as, determinations in the literature using oxygen as the metallicity tracer. The chemical analysis includes the sustraction of the nebular continuum and of the stellar continuum computed from simple stellar population synthesis grids. The $S^{+2}$ content is measured from the near infrared [SIII] $\lambda \lambda 9069 \AA, 9532 \AA$ lines, while an ICF $\left(S^{3+}\right)$ is proposed based on the $A r^{3+} / A r^{2+}$ fraction. Finally, we apply a multivariable linear regression using simultaneously oxygen, nitrogen and sulphur abundances for the same sample to determine the primordial helium abundance resulting in $Y_{P-O, N, S}=0.245 \pm 0.007$.
\end{abstract}

Key words: cosmology:primordial helium abundance; ISM:HII regions; sulphur abundance.

\section{INTRODUCTION}

The primordial light element abundances $\mathrm{D},{ }^{4} \mathrm{He}=\mathrm{Y}_{P},{ }^{3} \mathrm{He}$, ${ }^{7} \mathrm{Li}$ constitute a keystone in Big Bang cosmology along with the Cosmic Microwave Background (CMB) and the Universe expansion. A good agreement between observed abundances, and model parameters (such as $\eta$, the baryon to photon ratio, the number of neutrino families or the neutron half-life) enables a detailed definition of the Big Bang boundary conditions.

Almost twenty five years ago, precise measurements for the $D / H=3.40 \pm 0.25 \times 10^{-5}$ by Burles \& Tytler (1998) yielded a deuterium abundance almost a tenth of those previously reported (Songaila et al. 1994; Carswell et al. 1994). These observations caused a great commotion, since either higher $Y_{P}$ values, or new primordial nucleosynthesis models or non-standard physics would be required for consistency. The exploration of these possibilities also led to a thorough quantification of the systematic errors in the $Y_{P}$ determination. Overall, all these dedicated studies reached similar conclusions (Olive \& Skillman 2004; Fukugita \& Kawasaki

^ E-mail: vi.fernandez@inaoep.mx (Vital Fernández)
2006; Izotov et al. 2006; Rosales-Ortega 2006; Peimbert et al. 2007 a), providing values consistent with those of other cosmological parameters, even under the tighter WMAP and more recently Planck experiment limits (see Planck Collaboration et al. 2016).

The classical method to determine the primordial He abundance $Y_{P}$, proposed by Peimbert \& Torres-Peimbert (1974) and Lequeux et al. (1979), involves the extrapolation of the present helium abundance $Y$ down to zero metallicity. In practice, a single element, usually $O$, is used as metallicity tracer in ionized gas. The method is more reliable the lower the overall metal content of the gas to analyze. This situates low metallicity HII regions and local HII galaxies as the best objects for the job.

HII galaxies are compact, can be easily detected and among them the lowest metallicy galaxies are found. Their spectra are completely dominated by the current star formation episode and due to their intense gaseous emission spectra and weak underlying stellar continua, they can provide precise measurements of the He emission lines. In fact, in spectra with $E W(H \alpha)>200 \AA$, the underlying stellar continuum can hardly be noticed in the optical range, which minimizes this difficult to quantify source of systematic error in the derived He abundance. 
In these chemically unevolved objects, it is acceptable to assume a linear relation between the helium content and the oxygen abundance $\Delta Y / \Delta Z \approx \Delta Y / \Delta O$. Measuring the helium mass fraction and the $\mathrm{O}$ abundance of the gas for a given galaxy sample, the coefficients of the resulting linear regression are determined, i.e. $Y_{P}$ is the ordinate extrapolation to zero metals of the regression. Recently this method to determine $Y_{P}$ has been used in Aver et al. (2015) and Peimbert et al. (2016) yielding results $Y_{P}=0.2449 \pm 0.004$ and $Y_{P}=0.2446 \pm 0.0029$ respectively, consistent with Standard Big Bang Nucleosynthesis model predictions and with the value estimated by Planck Collaboration et al. (2016) $Y_{P}=0.24467 \pm 0.0002$. On the other hand, the result by Izotov et al. (2014), $Y_{P}=0.2551 \pm 0.0022$, is slightly outside the Planck collaboration value ${ }^{1}$. The three $Y_{P}$ determinations by Izotov et al. (2014); Aver et al. (2015); Peimbert et al. (2016) use the latest values of recombination coefficients for the HeI lines by Porter et al. (2012) and the associated corrigendum Porter et al. (2013).

As already mentioned, most of the research in this topic has used oxygen as the metallicity tracer in the $\mathrm{Y}$ vs. metal regression. Indeed, within the optical range, this element represents the obvious choice. However, it also presents clear difficulties. Firstly, while oxygen has the most intense lines in the optical range, the derivation of the electron temperature necessary for abundance determinations requires the detection and measurement of the weak auroral [OIII] $\lambda 4363 \AA$ line. This implies the selection of high excitation, high electron temperature objects for which a larger collisional excitation contribution to the hydrogen recombination lines is expected and this effect is challenging to quantify. On the other hand, due to the required high excitation, there is a maximum oxygen abundance of the objects to be used. This, in turn, decreases the baseline for the linear regression, increasing the $(\Delta Y / \Delta Z)$ gradient uncertainty.

Nitrogen was proposed as an alternative tracer by Pagel et al. (1992) who concluded that despite the nitrogen secondary production mechanism, a good concordance should be found between both metallicity tracers as long as the observed objects have a low nitrogen content $\left(N / H<6.6 \cdot 10^{-6}\right)$ and show no Wolf-Rayet features since the winds from these stars could cause local nitrogen pollution. This conclusion was confirmed by Rosales-Ortega (2006) using a sample of 71 low metallicity HII galaxies selected from the Sloan Digital Sky Survey (SDSS) who also found, unexpectedly, that the $Y_{P}$ determination using nitrogen was less uncertain for his sample and showed a better agreement with the WMAP experiment prediction than the corresponding value found using oxygen.

In this work we introduce the use of a third element, sulphur, as metallicity tracer. Although less abundant than oxygen, sulphur is also produced in massive stars through explosive nucleosynthesis and its yield should closely follow that of $\mathrm{O}$. Nebular $\mathrm{S} / \mathrm{H}$ abundances are therefore expected to follow those of $\mathrm{O} / \mathrm{H}$ and the $\mathrm{S} / \mathrm{O}$ ratio is expected to remain constant at about the solar value, $\log S / O \simeq-1.6$.

\footnotetext{
1 The Planck experiment provides four $\Lambda \mathrm{CDM} Y_{\boldsymbol{P}}$ estimations depending on the various data combinations. The value tabulated here represents an upper limit as considered by Peimbert et al. (2016)
}

Empirical tests exploring this have been performed confirming this ratio (Berg et al. 2015).

Sulphur abundances can be measured from near infrared observations where the $[S I I I] \lambda 9069 \AA$ and $[S I I I] \lambda 9532 \AA$ lines appear among the most intense features in the range. The region in the ionized nebulae where $S^{2+}$ originates practically covers the $O^{+}$and $O^{2+}$ one. The corresponding electron temperature can be well represented by $T_{e}[S I I I]$ derived from the line intensity ratio $I([S I I I] \lambda \lambda 9069 \AA, 9539 \AA) / I([S I I I] \lambda 6312)$ (e.g. Hägele et al. 2008 , and references therein). The use of the $S$ lines results in additional benefits compared to the other elements. Firstly, $[S I I I] \lambda 6312 \AA$ falls in a spectral region of high instrumental sensitivity. Secondly, these lines are weakly affected by underlying stellar population absorption. Furthermore, [SII] lines can be measured with respect to $H_{\alpha}$, while the near-infrared $[S I I I]$ lines can be measured with respect to nearby Paschen lines. This is useful to minimize reddening uncertainties and other calibration effects. Finally, and most important, the $S / H$ abundance can be measured by direct methods up to solar values. This is crucial in order to increase the dynamical range for the $\Delta Y / \Delta S$ gradient calculation, with respect to the $O / H$ and $N / H$ metallicity range. This would make the results less dependent on low $Y$ - metal poor galaxies, while increasing the sample to objects with higher $S / H$.

The paper has the following structure: Section 2 presents the observations of the selected HII galaxy sample and the data reduction. Section 3 describes the analysis of the data and the results attained. These results are discussed in Section 4. Finally, the conclusions of this work are summarized in Section 5.

\section{OBSERVATIONS AND DATA REDUCTION}

The HII galaxy sample was selected from the Sloan Digital Sky Survey (SDSS) to perform further ad-hoc observations. The current Data release, DR12, represents the final output from the SDSS-III survey (Ahn et al. (2014)). In addition to five photometric bands, spectroscopic data are included for over $6.7 \times 10^{5}$ stars, galaxies and quasars. The light, covering from $3800 \AA$ to $9200 \AA$ with a resolution power around $R=1800$ is received through a 3 -arcsec diameter fiber aperture. For low redshifts, this configuration enables an efficient selection of objects with strong emission lines, including the [SIII] $\lambda 9069 \AA$ line.

The primordial helium abundance determination requires the analysis of young, low metallicity and relatively high excitation nebulae, which contain a negligible or small amount of neutral helium. Additionally, a high signal-tonoise ratio is essential. High values of the hydrogen emission line equivalent widths guarantee this requirement while also selecting young objects with little underlying stellar population absorption. The galaxy sample selection has then been performed following the criteria:

- $E w(H \alpha)>200 \AA$

- $0.7<\sigma_{H \alpha}<3.5 \AA$

- $(S / N)_{H \alpha}>100$

- $[N I I] \lambda 6583 / H \alpha<0.2$

- $[O I I I] \lambda 4363 / H \beta \geq 0.1$ 
- $0.0007<z<0.2$

Finally, BPT diagrams (Baldwin et al. (1981)) were used in order to exclude AGN and LINERs. Special care was taken not to include objects with wide Wolf-Rayet features: the blue bump, around the HeII $44686 \AA$ line and the red bump, around the $C I V \lambda 5808 \AA$ line to avoid possible metal contamination.

\subsection{Sample description}

The spectra were acquired with the double arm Intermediate Dispersion Spectrograph and Imaging System (ISIS) attached to the $4.2 \mathrm{~m}$ William Herschel Telescope (WHT) at the Roque de los Muchachos Observatory during several observing runs between 2008 and 2015. The observations were taken using two different configurations.

In Configuration I, the R300B blue grating was centred at $\lambda_{c, \text { Blue }}=5950 \AA$ covering between $4300 \AA$ and $7600 \AA$ and the red $\mathrm{R} 316 \mathrm{R}$ one was centred at $\lambda_{c, \text { Red }}=8655 \AA$ to cover between $7260 \AA$ and $10050 \AA$. The 7500 dichroic was used in this set up.

Configuration II used the R300B grating covering between $3200 \AA$ and $5550 \AA$ and centred at $\lambda_{\text {c, Blue }}=4375 \AA$. The R310R grating in the red arm was centred at $\lambda_{c}$, Red $=$ $7615 \AA$ to cover between $5500 \AA$ and $9730 \AA$. The 5700 dichroic was used for this configuration.

For both setups, the EEV12 and REDPLUS detectors were used at the blue and red arm respectively. Both configurations provided a similar spectral dispersion of $0.86 \AA /$ pix with a pixel size close to 0.2 arcsec. The slit width was adjusted to the seeing with values between 1 and 1.5 arcsec. The observations were performed at small zenith distance and at parallactic angle to avoid effects of differential refraction in the UV-blue. In most cases these observations provided an adequate signal-to-noise for the analysis of weak lines such as $[S I I] \lambda \lambda 6717 \AA, 6731 \AA$, [OIII] $\lambda 4363 \AA$, $[$ ArIV] $\lambda 4740 \AA$ and $[S I I I] \lambda 6312 \AA$. Table 1 presents the observed sample. The first and second columns give the object name and the SDSS denomination respectively. The redshift is shown in column 3. The object coordinates are given in columns 4 and 5 and the instrumental configuration used is shown in column 6 .

Several bias and sky flat-field frames were obtained at both dusk and dawn. Additionally, a pair of flatfields and one calibration lamp exposure $(\mathrm{CuNe}+\mathrm{CuAr})$ were taken at each science position. The files were processed with IRAF $^{2}$ following standard procedures: bias subtraction, division by a normalized flatfield and wavelength calibration. The latter was accomplished using 20 to 40 lines. The spectra were flux calibrated and the edges between blue and red arms matched.To this purpose, at least two spectrophotometric standard stars were observed each night. This allowed a good flux calibration with an estimated precision of around $5 \%$.

NIR observations are affected by atmospheric telluric

2 IRAF: The Image Reduction and Analysis Facility is distributed by the National Optical Astronomy Observatories, which is operated by the Association of Universities for Research in Astronomy, Inc. (AURA) under cooperative agreement with the National Science Foundation (NSF). contamination. As an extra data reduction step, the object spectra were divided by a normalized featureless spectrum from a standard star observed with the same slit width as the object (Díaz et al. 1985). Fig. 1 illustrates this procedure for SDSS J024815.93-081716.5. The normalized spectrum belongs to the standard star Feige 34, whose absorption features have been clipped prior to the normalization operation. Unfortunately, the telluric observations could not be accomplished every night. For the objects whose $I([S I I I] \lambda 9532 \AA) / I([S I I I] \lambda 9069 \AA)$ ratio deviated considerably from the theoretical value $(2.47$ Hudson et al. 2012), only one of the lines was considered in our analysis choosing the one least affected by the atmospheric features. Two examples of the final reduced spectra are shown in Fig.2. The remaining sample spectra can be seen in the manuscript supporting material.

\section{ANALYSIS}

\subsection{Spectral components}

The emission lines represent the most distinctive features in HII galaxy spectra. The hydrogen and helium recombination lines are affected by the absorption lines from the ionizing stars spectrum, present at the same wavelengths. These absorptions can be very important for the fainter helium lines and must be taken into account in this element abundance calculation. Moreover, in the youngest HII regions, the hydrogen and helium nebular continua might contribute as much as the stellar continuum. The following subsections describe how these three components: nebular, stellar and emission line spectra are measured prior to undertaking any chemical analysis.

\subsection{Hydrogen and helium nebular continua}

Three distinct components contribute to the nebular continuum; they can be calculated from quantum physics principles. The free-free continuum is governed by Bremsstrahlung processes. As electrons travel within the ionized hydrogen or helium Coulomb's radius, they suffer a deflection and emit photons. These photons are of relatively low energy and hence this component contributes mainly in the infrared spectral region. This effect is calculated by Brown \& Mathews (1970) and Osterbrock (1974):

$$
\begin{aligned}
j_{v}, & =j_{v, H I}+j_{v H e I}+j_{v H e I I} \\
& =\frac{1}{4 \pi} n_{e}\left(n_{H^{+}} \gamma_{v}\left(H^{0}\right)+n_{H e^{+}} \gamma_{v}\left(H e^{0}\right)+\gamma_{v}\left(H e^{+}\right)\right)
\end{aligned}
$$

where $\left(j_{v}\right)$ is the continuum component emissivity, $\left(n_{e}\right)$ is the electron density, $\left(n_{H}, n_{H e}\right)$ are the hydrogen and helium ion densities and $\left(\gamma_{v}, f f\right)$ is the continuous emission coefficient. In the case of the free-free component, the latter coefficient can be calculated from the following relation, in which the quantum effects can be neglected, $\left(g_{f f}\left(T_{e}, Z, v\right) \approx 1\right)$ for the electron temperatures commonly encountered in HII regions:

$$
\gamma_{\nu, f f}=\text { const. }\left(\frac{\pi h v_{0}}{3 k T_{e}}\right)^{1 / 2} e^{-h v / k T}
$$

where $v_{0}$ denotes the threshold frequency for the ionization of $H^{0}\left(h v_{0}=13.6 \mathrm{eV}\right)$.

The free-bound emission, to which both hydrogen and helium atoms contribute, is produced from the electron transitions from 

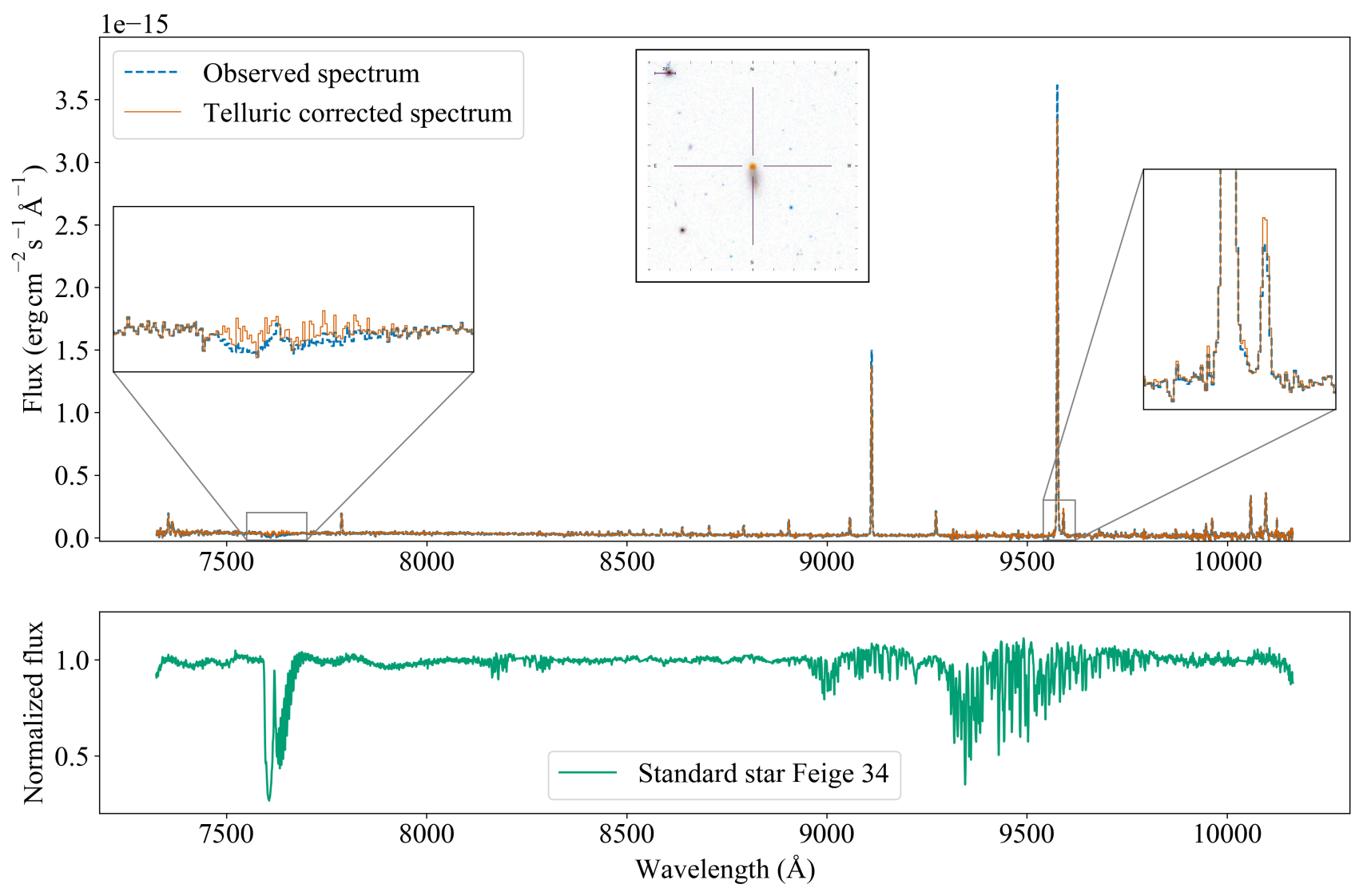

Figure 1. Telluric correction applied to SDSSJ024815.93-081716.5 red arm spectrum. The calibrated object is divided by the normalized spectrum from a standard star whose absorption features are artificially removed. The stellar spectrum was acquired with the same slit width as the science objects.

free to bound states (recombination). This process is responsible for the Paschen and Balmer jumps. Computing $\gamma_{v, f b}$, requires to add up all the recombination to states of principal quantum number (n) (e.g. Ercolano \& Storey 2006). In their hydrogenic codes, convergence is obtained by considering principal quantum levels up to $n=200$ for hydrogen and $n=350$ for helium. Their results are presented in tabular form, where $\gamma_{v, f b}$ is parametrised as a function of temperature and wavelength.

Finally, in the ultraviolet region, the main nebular contribution comes from the hydrogen $2 s \rightarrow 1 s$ decay, which produces a photon pair. Thereby, this emission is commonly referred to as 2photon continuum. The continuous emission coefficient $\left(\gamma_{v}, 2 q\right)$ is characterized by the parameter $g_{v}\left(T_{e}\right)$ (Nussbaumer \& Schmutz 1984) following the scheme presented by Osterbrock (1974):

$\gamma_{v, 2 q}=\frac{\alpha_{e f f} g_{v}}{1+q_{2} / A_{2 q}}$

where $\left(A_{2 q}=8.23 s\right)$ is the transition probability for the photon decay and $\left(\alpha_{e f f}\right)$ and $\left(q_{2}\right)$ are the effective recombination coefficient and collisional transition rate for protons and electrons respectively. These parameters are determined from the fits of Mollá et al. (2009):

$$
\begin{aligned}
\alpha_{e f f} & =0.647 \cdot 10^{-10} \cdot T_{e}^{-0.722} \\
q_{2} & =5.92 \cdot 10^{-4}-6.1 \cdot 10^{-9} T_{e}
\end{aligned}
$$

Once $\left(\gamma_{v}\right)$ has been tabulated for each process and ion, the total nebular emissivity can be calculated from the $\mathrm{HeI}$ and $\mathrm{HeII}$ fractions. The final hydrogen and helium components are computed as:

$j_{v}=\frac{n_{e} n\left(H^{+}\right)}{4 \pi}\left(\gamma_{v}\left(H^{0}\right)+\frac{n\left(H e^{+}\right)}{n\left(H^{0}\right)} \gamma_{v}\left(H e^{0}\right)+\frac{n\left(H e^{2+}\right)}{n\left(H^{+}\right)} \gamma_{v}\left(H e^{+}\right)\right)$

where,

$$
\begin{aligned}
\gamma_{v}\left(H^{0}\right) & =\gamma_{v, f f}\left(H^{0}\right)+\gamma_{v, f b}\left(H^{0}\right)+\gamma_{v, 2 q}\left(H^{0}\right) \\
\gamma_{v}\left(H e^{0}\right) & =\gamma_{v, f f}\left(H e^{0}\right)+\gamma_{v, f b}\left(H^{0}\right) \\
\gamma_{v}\left(H e^{+}\right) & =\gamma_{v, f f}\left(H e^{+}\right)+\gamma_{v, f b}\left(H^{+}\right)
\end{aligned}
$$

Up to this point, the nebular continuum calculation requires some estimates of the electron temperature and of $\mathrm{He}^{0}$ and $\mathrm{He}^{+}$ abundances. A characteristic low ionization temperature from the [SIII] lines was used. This is a reasonable assumption as the twice ionized sulphur population covers a large percentage of the HII region volume.

Translating emissivity to flux for a given object would require accurate knowledge of both distance and density. Fortunately, a more feasible approach is proposed by Zanstra (1931) to estimate the ultraviolet continuum produced by the ionizing stars from the recombination lines intensity. In our case, we compare the emissivity from a recombination line and the nebular continuum. From first principles, a line emission luminosity, e.g. $H \boldsymbol{\beta}$, is given by:

$$
\begin{aligned}
L(H \beta) & =h v_{H \beta} \int_{0}^{r_{1}} 4 \pi j_{H \beta} d V \\
& =h v_{H \beta} \int_{0}^{r_{1}} 4 \pi n_{p} n_{e} \alpha_{H \beta}^{e f f} d V
\end{aligned}
$$


Table 1. Sample observations. Column (1) gives an existing object name (otherwise an order name ad-hoc for this paper). Two regions (A1 and A2) were observed in MRK36.

\begin{tabular}{|c|c|c|c|c|c|}
\hline Local reference & SDSS reference & $\mathrm{z}$ & RA (hh:':") & DEC (deg:':") & ISIS configuration \\
\hline FTDTR-1 & SDSSJ012534.19+075924.5 & 0.009593 & $1: 25: 34.212$ & $+07: 59: 24.59$ & $\mathrm{I}$ \\
\hline IZw18 & SDSSJ093402.02+551427.8 & 0.002614 & 9:34:02.162 & $+55: 14: 22.34$ & I \\
\hline MRK36-A1 & SDSSJ110458.30+290816.6 & 0.002190 & 11:04:58.564 & $+29: 08: 14.89$ & I \\
\hline MRK36-A2 & SDSSJ110458.30+290816.6 & 0.002202 & 11:04:58.564 & $+29: 08: 14.89$ & I \\
\hline MRK475 & SDSSJ143905.46+364821.8 & 0.002024 & 14:39:05.466 & $+36: 48: 21.97$ & I \\
\hline FTDTR-2 & SDSSJ171236.63+321633.4 & 0.01203 & $17: 12: 37.071$ & $+32: 16: 29.52$ & I \\
\hline IZw70 & SDSSJ145056.56+353419.5 & 0.003953 & 14:50:56.801 & $+35: 34: 16.74$ & I \\
\hline MRK689 & SDSSJ153619.43+304056.4 & 0.005793 & 15:36:19.439 & $+30: 40: 56.40$ & I \\
\hline MRK67 & SDSSJ134156.47+303109.6 & 0.003316 & $13: 41: 56.956$ & $+30: 31: 05.91$ & I \\
\hline FTDTR-3 & SDSSJ122129.18+282208.3 & 0.008695 & $12: 21: 29.178$ & $+28: 22: 08.44$ & I \\
\hline SHOC022 & SDSSJ003218.59+150014.1 & 0.01793 & $0: 32: 19.337$ & $+15: 00: 02.48$ & I \\
\hline FTDTR-4 & SDSSJ081334.17+313252.1 & 0.01959 & $8: 13: 33.374$ & $+31: 33: 10.29$ & I \\
\hline SHOC220 & SDSSJ084029.91+470710.2 & 0.04210 & $8: 40: 28.462$ & $+47: 07: 19.92$ & I \\
\hline FTDTR-5 & SDSSJ100348.66+450457.6 & 0.009252 & 10:03:47.356 & $+45: 05: 06.37$ & I \\
\hline FTDTR-6 & SDSSJ024815.93-081716.5 & 0.004528 & $2: 48: 16.920$ & $-08: 17: 15.12$ & I \\
\hline FTDTR-7 & SDSSJ082334.84+031315.6 & 0.009622 & $8: 23: 35.322$ & $+03: 13: 17.57$ & I \\
\hline MRK627 & SDSSJ084634.40+362620.8 & 0.01059 & $8: 46: 33.891$ & $+36: 26: 33.81$ & I \\
\hline SHOC592 & SDSSJ212332.71-074831.0 & 0.02805 & $21: 23: 32.225$ & $-07: 48: 27.21$ & II \\
\hline PHL293B & SDSSJ223036.79-000636.9 & 0.005138 & $22: 30: 36.429$ & $-00: 06: 33.92$ & II \\
\hline SHOC588 & SDSSJ211527.07-075951.3 & 0.02837 & $21: 15: 27.200$ & $-07: 59: 54.95$ & II \\
\hline SHOC036 & SDSSJ005147.29+000940.0 & 0.03738 & $0: 51: 47.394$ & $+00: 09: 24.05$ & II \\
\hline SHOC575 & SDSSJ172906.55+565319.3 & 0.01601 & 17:29:07.491 & $+56: 53: 12.35$ & II \\
\hline SHOC579 & SDSSJ173501.25+570308.5 & 0.04739 & $17: 35: 00.687$ & $+57: 02: 52.42$ & II \\
\hline FTDTR-8 & SDSSJ100348.66+450457.6 & 0.009251 & 10:03:48.766 & $+45: 04: 53.69$ & I \\
\hline SHOC263 & SDSSJ093813.49+542825.1 & 0.1022 & 9:38:13.761 & $+54: 28: 22.20$ & I \\
\hline FTDTR-9 & SDSSJ100746.51+025228.3 & 0.02330 & 10:07:46.500 & $+02: 52: 27.98$ & I \\
\hline FTDTR-10 & SDSSJ102429.25+052450.9 & 0.03313 & $10: 24: 29.237$ & $+05: 24: 50.71$ & I \\
\hline
\end{tabular}

where $\left(\alpha_{H \beta}^{e f f}\right)$ is the effective recombination coefficient for the $H \beta$ emission line which is tabulated in Pequignot et al. (1991).

To obtain the luminosity from the nebular continuum we integrate the emissivity $j_{v}$, over the ionized volume:

$$
\begin{aligned}
L_{\nu} & =\int_{0}^{r_{1}} 4 \pi j_{\nu} d V=\int_{0}^{r_{1}} 4 \pi\left(\frac{1}{4 \pi} n_{p} n_{e} \gamma_{\nu}\right) d V \\
& =\int_{0}^{r_{1}} n_{p} n_{e} \gamma_{\nu} d V
\end{aligned}
$$

Dividing eq. 13 by eq.10 we obtain:

$$
\frac{L_{v}}{L(H \beta)}=\frac{\gamma_{v} \int_{0}^{r_{1}} n_{p} n_{e} d V}{h v_{H \beta} \alpha_{H \beta}^{e f f} \int_{0}^{r_{1}} 4 \pi n_{p} n_{e} d V}
$$

which can be approximated by:

$$
\frac{F_{N e b, v}}{F(H \beta)} \approx \frac{\gamma_{v}}{\alpha_{H \beta}^{e f f} \cdot h v_{H \beta}}
$$

This simplification is valid as long as the $\left(\gamma_{\nu} / \alpha_{H \beta}^{e f f}\right)$ ratio remains constant with temperature. This is a reasonable assumption for the physical conditions commonly encountered in HII galaxies.

\subsection{Underlying stellar continuum}

The observed hydrogen and helium lines are the combination of the emission lines from the ionized gas and the absorption lines from the ionizing stars atmosphere. In order to obtain a pure emission line spectrum the subtraction of the stellar contribution is mandatory. This can be accomplished with the help of stellar population synthesis models. Here we used the STARLIGHT synthesis code (Cid Fernandes et al. 2005). In this code, we used a Single Stellar Population (SSP) library with ages, between $1 \mathrm{Myr}$ and $17 G y r$, and metallicities, between $Z_{\odot} / 200$ and $1.5 \cdot Z_{\odot}$. Suitable emission line masks were manually selected for each observed spectrum as described in the following section. Additionally, the first and final $50 \AA$ of the observed spectra were also masked. The spectra were re-sampled to $1 \AA /$ pixel as required by the code.

In our analysis, the observations were reduced without taking into account the error propagation. Consequently, the error per pixel is not quantified. As an alternative, STARLIGHT computes a global value from the flux rms used during the normalization. In our case, this flux window is between $4750 \AA$ and $4840 \AA$. Initially the minimum visual extinction was set to zero and the default maximum velocity dispersion was set to each galaxy $\sigma_{\mathrm{CaII}}$ or $\sigma_{[O I I I]}$ value, the velocity dispersion measured from the width of the near-IR CaII triplet absorptions or from the [OIII] emission lines.

\subsection{Line emission}

Once the nebular and stellar continua are removed from the observed spectrum, the pure emission line fluxes can be measured. A graphical interface was designed to inspect the spectral features in the relevant wavelength range. The detected emission line along with two adjacent continuum regions of at least $20 \AA$ were manually selected and a linear continuum fit was performed. The integrated line flux was then measured. The uncertainty from each line flux and equivalent width was estimated using a bootstrap algorithm: a set of random realizations of every line pixel was generated using data poissonian $1 \sigma$ uncertainty from the continua adjacent to the line. At each iteration, the area under the line is measured and a Gaussian fit performed. This generates a distribution for the line flux and Gaussian parameters (peak intensity, central wavelength and standard deviation) from which their $1 \sigma$ uncertainty is determined.

A similar procedure was applied to blended lines, where their flux is determined assuming a combination of Gaussian curves. 

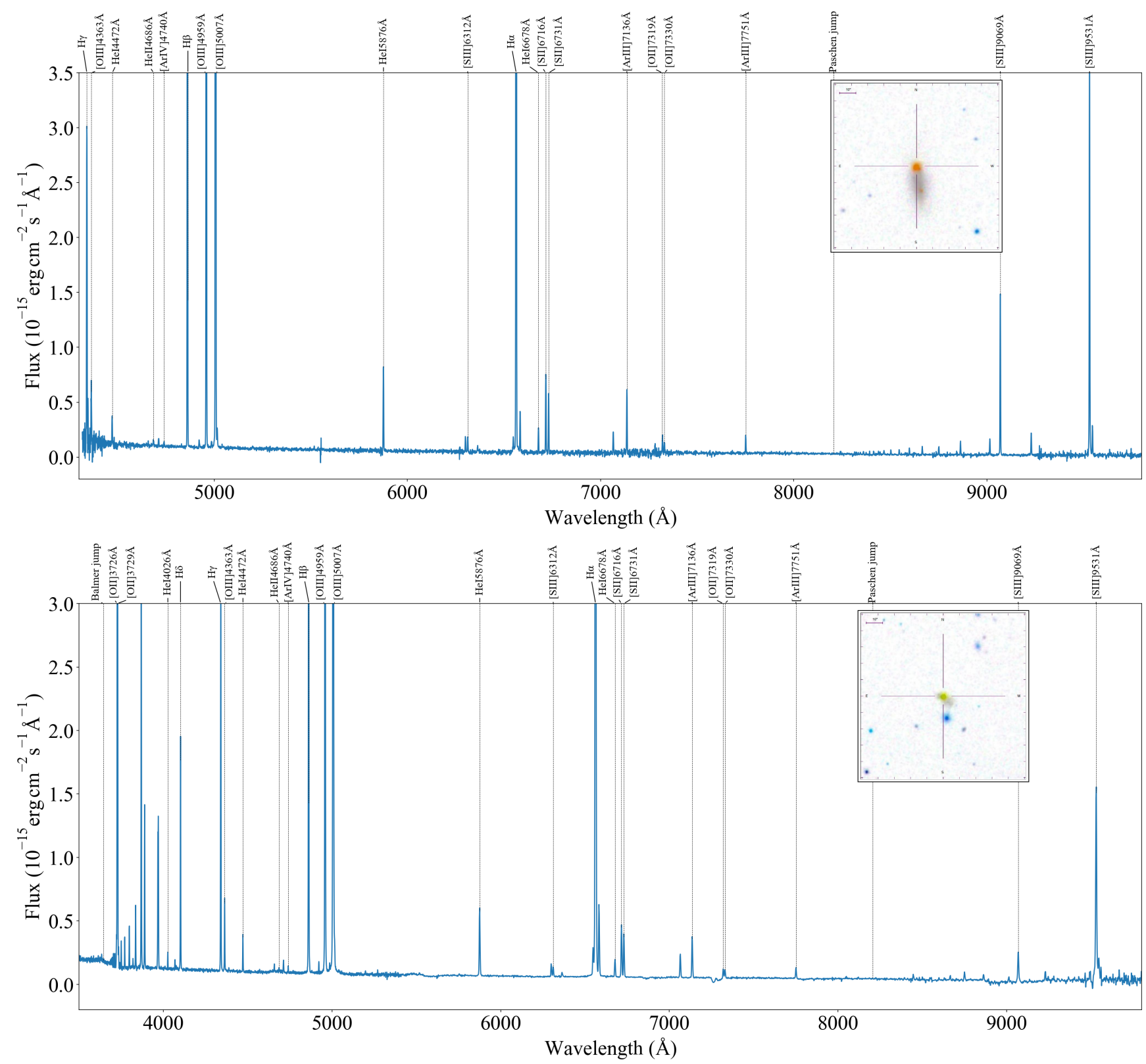

Figure 2. Sample of reduced spectra for objects FTDR6 (top, configuration I) and SHOC579 (bottom, Configuration II). Relevant lines are labelled. The object images belong to the SDSS database.

Extra care was given to the $[N I I] \lambda \lambda 6548 \AA, 6583 \AA$ and $H \alpha$ region. Even though our resolution is high enough to separate all three lines, for some objects $H \alpha$ has a broad component that contaminates the $[N I I]$ emission. The intensity and shape of this component are commonly explained by gas inflows/outflows and hence it changes from object to object. The following strategy provided the best output:

(i) Three Gaussians were fitted for $[N I I] 6548 \AA, H \alpha$, [NII] $6583 \AA$ narrow components with the following constraints: the central wavelength of the narrow features must be within $\pm 2 \AA$ of the expected value; both hydrogen and nitrogen emissions are assumed to share the same $\sigma$; and the $[N I I] 6583 \AA /[N I I] 6548 \AA$ flux ratio must be 2.94 [assuming the $N^{+}$atomic data from Tayal (2011), Wiese et al. (1996) and Galavís et al. (1997)].

(ii) The fitted narrow emission is removed from the spectrum leaving only the $H \boldsymbol{\alpha}$ broad component, which is fitted with a Gaussian curve.

(iii) In the last step, both the narrow and wide $H \alpha$ components are fitted using constraints from the previous fits: the wide component central wavelength is fixed from the value obtained on the second fit. Additionally, its peak flux is also contained within $\pm 10 \%$ the value obtained in the previous fitting. The narrow components have the same constraints as those described for the first fitting.

\subsection{Chemical analysis}

Table 2 includes the emission line data for two example HII galaxies, one for each configuration. Column (1) lists the line and wavelength. Column (2) provides the equivalent width. Column (3) and (4) give the line flux and the reddening-corrected line inten- 
Table 2. Relative line intensities with respect to $H \beta$ and multiplied by 1000 for objects FTDTR-6 (Configuration I) and SHOC579 (Configuration II). Nebular and stellar continua have been removed.

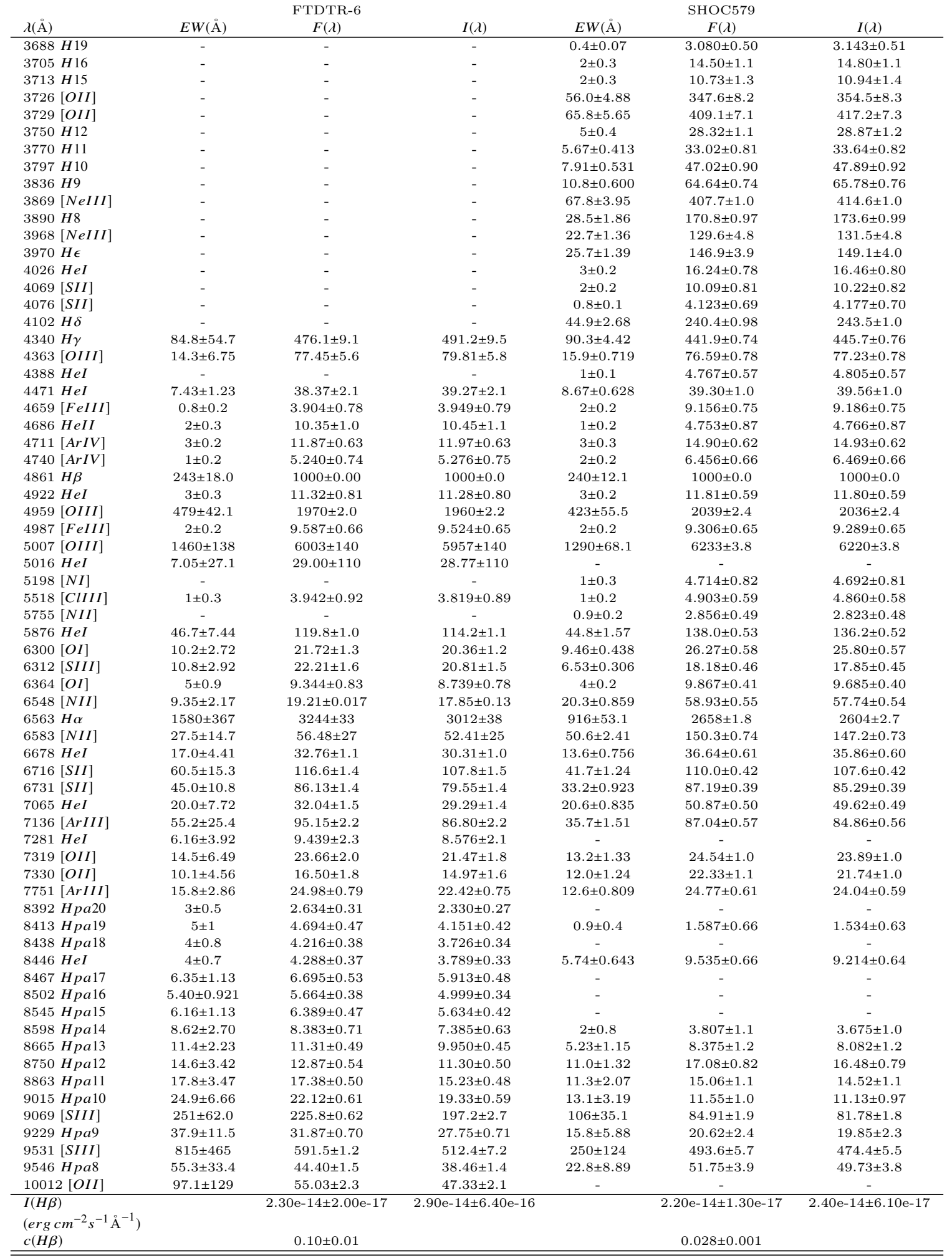

sity in units of $H \beta=1000$. Finally, at the foot of the table, the raw and reddening corrected $H \beta$ fluxes for both galaxies are given in $\operatorname{erg~} \mathrm{cm}^{-2} \mathrm{~s}^{-1} \AA^{-1}$. These values have been corrected for the underlying stellar continuum. The remaining tables for the complete sample are available online. The emission lines were corrected for reddening. The $c(H \beta)$ coefficient, determined canonically from the Balmer decrement is also given in the table. We considered appropriate for these young starforming bursts the "LMC average" reddening curve from Gordon et al. (2003) with $R_{V}=3.4$. The recombination coefficients are calculated using PyNeb (see 
Luridiana et al. 2015, among others) with the atomic data of Storey \& Hummer (1995). The Paschen series was not included in the $c(H \beta)$ calculation as the simple stellar populations used reach only up to $7000 \AA$ as already mentioned.

The abundances of $\mathrm{He}, \mathrm{S}, \mathrm{O}$ and $\mathrm{N}$ are calculated from the reddening corrected emission line fluxes also using PyNeb. The tools provided by this library were scripted into a Monte Carlo algorithm to propagate the uncertainty in the emission line fluxes to the electron temperatures and densities, and finally, to the chemical abundances. The abundances were determined following these steps:

(i) PyNeb calculates simultaneously $n_{e}$ and $T_{e}$ using suitable density and temperature diagnostic lines. Lines which arise from similar excitation energies for the former and the ratio of lines with different excitation energies to estimate the latter as usual. The $S^{+}$density determined from the $[S I I] \lambda \lambda 6716 \AA$, $6731 \AA$ lines diagnostic is adopted for all species. This diagnostic, however, starts to loose sensitivity at densities below $100 \mathrm{~cm}^{-3}$. For objects where the density calculated from these diagnostic was below $75 \mathrm{~cm}^{-3}$ the value was set to $50 \pm 25 \mathrm{~cm}^{-3}$.

(ii) We considered a three zone model for the electron temperature: a high ionization zone where ions like $\mathrm{O}^{2+}, \mathrm{Ne}^{2+}$ are produced; a lower ionization zone where ions like $\mathrm{O}^{+}, \mathrm{Ne}^{+}, \mathrm{N}^{+}$, $S^{+}$are formed and a third intermediate ionization zone which partially overlaps the previous two ones, where ions like $S^{2+}$ and $A r^{2+}$ are formed. The different temperatures that characterize each ion population are further discussed in the following subsections.

(iii) For the majority of the objects, similar temperature precision was attained for $T_{e}[S I I I]$ and $T_{e}[O I I I]$. This can be noticed in Fig. 3, where both temperatures are plotted for the observed galaxies. For some objects though, the uncertainty is larger for one of the temperatures. If this is the $T_{e}[O I I I]$, it usually corresponds to data obtained with instrument configuration $\mathrm{I}$, in which the $[O I I I] \lambda 4363 \AA$ line is very close to the blue edge of the spectrum, hence it is noisier. The cases with larger error in $T_{e}[S I I I]$ are those that show important telluric contamination around the $S^{2+}$ lines, where the continuum was extracted. Still, as seen in the figure, the correspondence is quite good as expected (Garnett 1992). Consequently, it is possible to replace the higher-uncertainty temperature using the linear fitting, while propagating the lower uncertainty. We used eq. 16, taken from Pérez-Montero \& Díaz (2005), who use the same atomic parameters as we are using in this work for the sulphur and oxygen ions:

$$
t_{e}[O I I I]=1.0807 \times t_{e}[S I I I]-0.0846
$$

Additional literature fits are also plotted in Fig. 3, as it can be appreciated, they are all very similar in the $10000-16000 \mathrm{~K}$ range.

(iv) The ionic abundances are calculated from the most intense line (if more than one is available their intensities are added up).

(v) For the error propagation we used a bootstrap algorithm: for each line a one thousand length array is generated. The fluxes in this array come from a normal distribution defined by the mean line intensity and its uncertainty.

(vi) Since the bootstrap algorithm assumes a normal distribution for the generated flux arrays, some diagnostic ratios may lie beyond the physical boundaries. In these cases, PyNeb generates a non numerical entry. These values are systematically removed at each step and are replaced with random values generated from a normal distribution of the valid entries.

The references for the atomic data used in these calculations are listed in Table 3.

\subsubsection{Helium:}

The helium spectrum shows several lines within the observed range. Clearly, the greater the number of lines considered, the

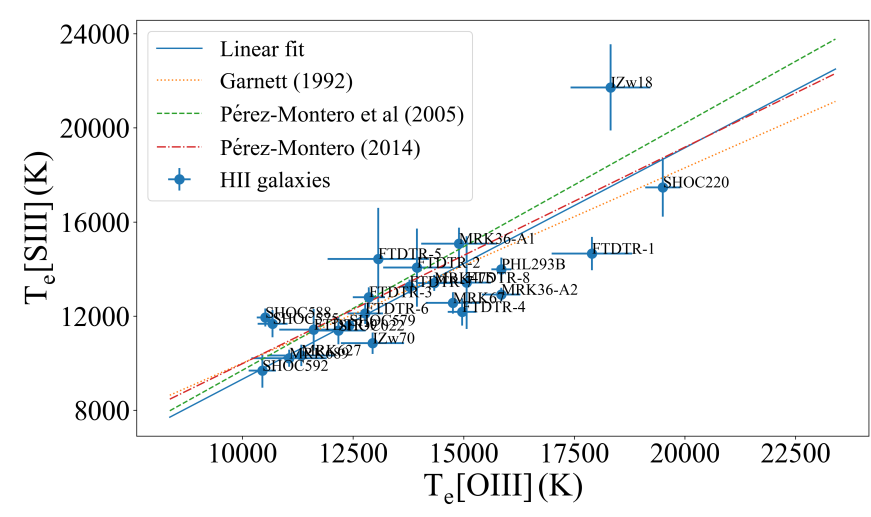

Figure 3. $\mathrm{T}_{e}[S I I I]$ vs. $\mathrm{T}_{e}[O I I I]$. The blue solid line is the fit to the data. The dotted orange line represents the models prediction by Garnett (1992). The green and red dashed lines correspond to the photoionization models from Pérez-Montero et al. (2005) and Pérez-Montero (2014) respectively.

most robust the analysis. However, not all the lines share the same dependence on $T_{e}$ and $n_{e}$ and some are faint. Moreover, not all lines are evenly affected by external physical phenomena e.g. bluer lines are more susceptible to underlying stellar absorption and reddening. (e.g. Aver et al. 2015, and references therein) solved simultaneously for the six physical parameters contributing to the helium line intensity. In the present study these are quantified separately. The stellar absorption is corrected from the SSPs fit once the nebular continuum has been removed from the observed spectrum. The reddening coefficient $(c(H \beta))$ is calculated from the Balmer decrement. The collisional effect on the helium lines is already included in the emissivities (Porter et al. 2013). Finally, we assumed $T(\mathrm{HeI})=T_{e}[\mathrm{OIII}]=T_{h i g h}$ and $n_{e}(\mathrm{HeI})=n_{e}[\mathrm{SII}]$. At this point the abundance is calculated using $\chi^{2}$ fitting over the set of HeI lines:

$\chi^{2}=\sum_{i=1}^{n} \frac{\left(I_{H e \lambda, i, o b s}-I_{H e \lambda, i, t h e o}\right)^{2}}{\sigma_{H e \lambda, i}^{2}}$

where $\left(I_{H e \lambda, i}\right)$ corresponds to a particular $\mathrm{HeI}$ line intensity and $\left(\sigma_{i}\right)$ to its uncertainty (both normalized to $H \beta=1000$ ). The theoretical line intensity is computed as:

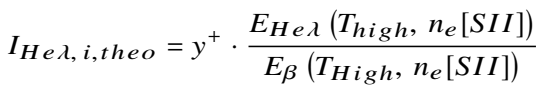

This fit is repeated in a one thousand bootstrap algorithm, in which $T_{e}$ and $n_{e}$ values are randomly generated from the measured values assuming a Gaussian distribution. The algorithm output is itself a distribution which provides $\mathrm{He}^{+}$mean value and uncertainty. The code uses the HeI $\lambda 4471 \AA$, $5876 \AA$, $6678 \AA$ lines to perform the fit.

A similar approach is used to calculate the $\mathrm{He}^{2+}$ abundance if the HeII $44686 \AA$ line is observed. The previous assumptions imply that the HeI and HeII emission are produced in the high ionization region. This is an acceptable assumption for HeII as can be seen by using photoionization models. For HeI, Peimbert and colaborators (Peimbert et al. 2002) analysed the possible bias introduced on the primordial helium determination by assuming $T_{e}(\mathrm{HeI})=T_{e}(\mathrm{OIII})$. However, as discussed by Aver et al. (2010) and Aver et al. (2015), a large degeneracy in the temperature exists when calculating helium abundance, quite possibly enhanced by temperature fluctuations (Luridiana et al. 2003). To overcome this degeneracy, Aver and collaborators have used a temperature prior based on the measured $\mathrm{T}_{e}([O I I I])$ to fit the model parameters. Still, the mean uncertainty in the helium temperature for their flagged sample is $\Delta T_{e}\left(H_{e}\right) \approx 2200 \mathrm{~K}$. The approach proposed here represents a simplification that consists on using $T_{e}([O I I I])$ 
Table 3. Atomic data sources

\begin{tabular}{ccc}
\hline Ion & Atomic data \\
\hline \hline$H$ & Storey \& Hummer (1995) \\
\hline $\boldsymbol{H} e$ & Porter et al. (2013) \\
\hline $\boldsymbol{H} \boldsymbol{e}^{+}$ & Storey \& Hummer (1995) \\
\hline \hline Ion & Collision Strengths & Transition probabilities \\
\hline \hline $\boldsymbol{O}^{+}$ & Pradhan et al. (2006); Tayal (2007) & Zeippen (1982); Wiese et al. (1996) \\
\hline $\boldsymbol{O}^{+2}$ & Aggarwal \& Keenan (2000) & Storey \& Zeippen (2000); Wiese et al. (1996) \\
\hline$N^{+}$ & Tayal (2011) & Wiese et al. (1996); Galavís et al. (1997) \\
\hline$S^{+}$ & Tayal \& Zatsarinny (2010) & Podobedova et al. (2009) \\
\hline$S^{+2}$ & Hudson et al. (2012) & Podobedova et al. (2009) \\
\hline$S^{+3}$ & Tayal (2000) & Dufton et al. (1982); Johnson et al. (1986) \\
\hline$A r^{+2}$ & Galavís et al. (1995) & Kaufman \& Sugar (1986); Galavís et al. (1995) \\
\hline$A r^{+3}$ & Ramsbottom \& Bell (1997) & Mendoza \& Zeippen (1982) \\
\hline
\end{tabular}

to generate a distribution of values to compute the $T_{e}(\mathrm{HeI})$. A more rigorous estimate of $T_{e}(\mathrm{HeI})$ is left for a forthcoming paper (Fernández et al, in preparation). The helium lines observed in both our instrumental setups are shown in Table 2. The total He abundance is calculated as:

$\frac{H e}{H}=\frac{H e^{+}}{H^{+}}+\frac{H e^{2+}}{H^{+}}$

\subsubsection{Sulphur}

$n_{e}\left(S^{+}\right)$is commonly assumed in the literature to characterize HII regions whose densities are below the critical value for collisional deexcitation $\left(\sim 10^{4}\right)$. Regarding the electron temperature, we assume $T_{e}\left(S^{+}\right) \approx T_{e}\left(S^{2+}\right)=T_{e}[S I I I]$. Justification for this model can be found in the ionization structure diagrams, such as those introduced by Garnett (1992). Fig.4 was generated using the data from the evolutionary synthesis models developed by Martín-Manjón et al. (2010) within the PopStar project. The radiation spectrum along with the ionizing photons, inner radius and gas metallicity were used as the input conditions in Cloudy 17.01 (Ferland et al. 2017). This enabled us to simulate a wide range of starforming regions. The model grid is characterized by $\log ($ age $)=5.0-10.0, Z_{\text {gas }}=0.0004-0.05$, Mass $=12000-200000 M_{\odot}, \log \left(Z_{\star}\right)=-2.1$ and $n_{e}=100 \mathrm{~cm}^{-3}$.

Fig. 4 displays the ionization structure from two of the models: oxygen and sulphur for $10^{5} M_{\odot}$ and $2 \cdot 10^{5} M_{\odot}$ cluster masses. A sharp transition between $S^{+}$and $S^{2+}$ ionization states can be seen. Both ions are observed in the transition zone and a common electron temperature may be assumed. Henceforth, this temperature is referred to as $T_{l o w}$ and the sulphur abundance is calculated as:

$$
\begin{aligned}
n_{e}\left(S^{+}\right)=n_{e}[S I I] & =f\left(\frac{I([S I I] 6716 \AA)}{I([S I I] 6731 \AA)}\right) \\
T_{\text {low }}=T_{e}[S I I I] & =f\left(\frac{I([S I I I] 6312 \AA)}{I([S I I I] 9069 \AA+[S I I I] 9532 \AA)}\right) \\
\frac{S^{+}}{H^{+}} & =f\left(\sum_{i} I_{i}([S I I]), T_{\text {low }}, n_{e}[S I I]\right) \\
\frac{S^{2+}}{H^{+}} & =f\left(\sum_{i} I_{i}([S I I I]), T_{\text {low }}, n_{e}[S I I]\right)
\end{aligned}
$$

Due to the low ionization potential for sulphur, the neutral $\mathrm{S}$ fraction can be neglected. However, this may not be true for the $S^{3+}$ population. A direct $S^{3+}$ determination would require the flux from the infrared $[S I V] 10.51 \mu \mathrm{m}$ line. As an alternative we propose an individualized ICF (Ionization Correction Factor) based on argon. The $A r^{+2} / A r^{+3}$ vs. $S^{2+} / S^{3+}$ relation is shown in
Fig. 5 for the models grid previously described. A linear relation for both elements at low gas metallicity is seen in the figure. A linear fitting gives:

$$
\log \left(\frac{A r^{2+}}{A r^{3+}}\right)=a \cdot \log \left(\frac{S^{2+}}{S^{3+}}\right)+b
$$

$a=1.162 \pm 0.006 ; b=0.05 \pm 0.01$

This ICF is suitable for optical observations as transitions from the $\mathrm{Ar}^{+3}$ ion are observable in the optical range. The argon ionic ratio is calculated as:

$$
\begin{aligned}
& \frac{A r^{2+}}{H^{+}}=f\left(\sum_{i} I_{i}([A r I I I]), T_{l o w}, n_{e}[S I I]\right) \\
& \frac{A r^{3+}}{H^{+}}=f\left(\sum_{i} I_{i}([A r I V]), T_{\text {high }}, n_{e}[S I I]\right)
\end{aligned}
$$

where $T_{\text {high }}$ is the temperature for the high ionization region and the $T([O I I I])$ temperature is used to characterize it. Its calculation is detailed in section 3.5.3. The total sulphur abundance is defined as:

$\frac{S}{H}=\left(\frac{S^{+}}{H^{+}}+\frac{S^{2+}}{H^{+}}\right) \cdot I C F\left(S^{3+}\right)$

We set $I C F\left(S^{3+}\right)=1$ for objects where the $A r^{3+}$ lines are not observed, and consequently assumed $S^{3+} / H^{+} \approx 0$.

\subsubsection{Oxygen}

It is commonly accepted that the $\mathrm{O}^{2+}$ ion characterizes the temperature of the highly ionized gas in these metal poor star forming galaxies. This assumes $T_{e}\left(O^{2+}\right) \approx T_{e}[O I I I]$. Contrary to sulphur discussed above, $T_{e}\left(O^{+}\right) \nsim T_{e}\left(O^{2+}\right)$. Indeed, the $O^{+}$ ions are situated in a lower ionization region with a lower mean temperature. For this zone it is possible to adopt $T_{e}[O I I] \approx$ $T_{e}[S I I I]$ Hägele et al. $(2006,2008)$. To calculate the ionic abundances, the $O^{2+}$ fraction can be accurately measured from the [OIII] $\lambda \lambda 4959 \AA$, $5007 \AA$ lines. Ideally the $O^{+}$abundance should be measured from the strong $[O I I] \lambda \lambda 3726 \AA, 3729 \AA$ doublet. Unfortunately, these lines are not available for objects observed with our configuration I (see Table 1). For these objects we use the $[O I I] \lambda \lambda 7319 \AA, 7330 \AA$ weaker auroral lines doublet. Although these lines have been used in the literature to calculate the $\mathrm{O}^{+}$abundance (see Kniazev et al. 2003; Pilyugin et al. 2006; Sánchez Almeida et al. 2016), they have also been reported to increase the dispersion on physical parameters such as the $T_{e}[O I I]$ as described by Kennicutt et al. (2003). These authors argue that one phenomenon contributing to these deviations is the recombi- 


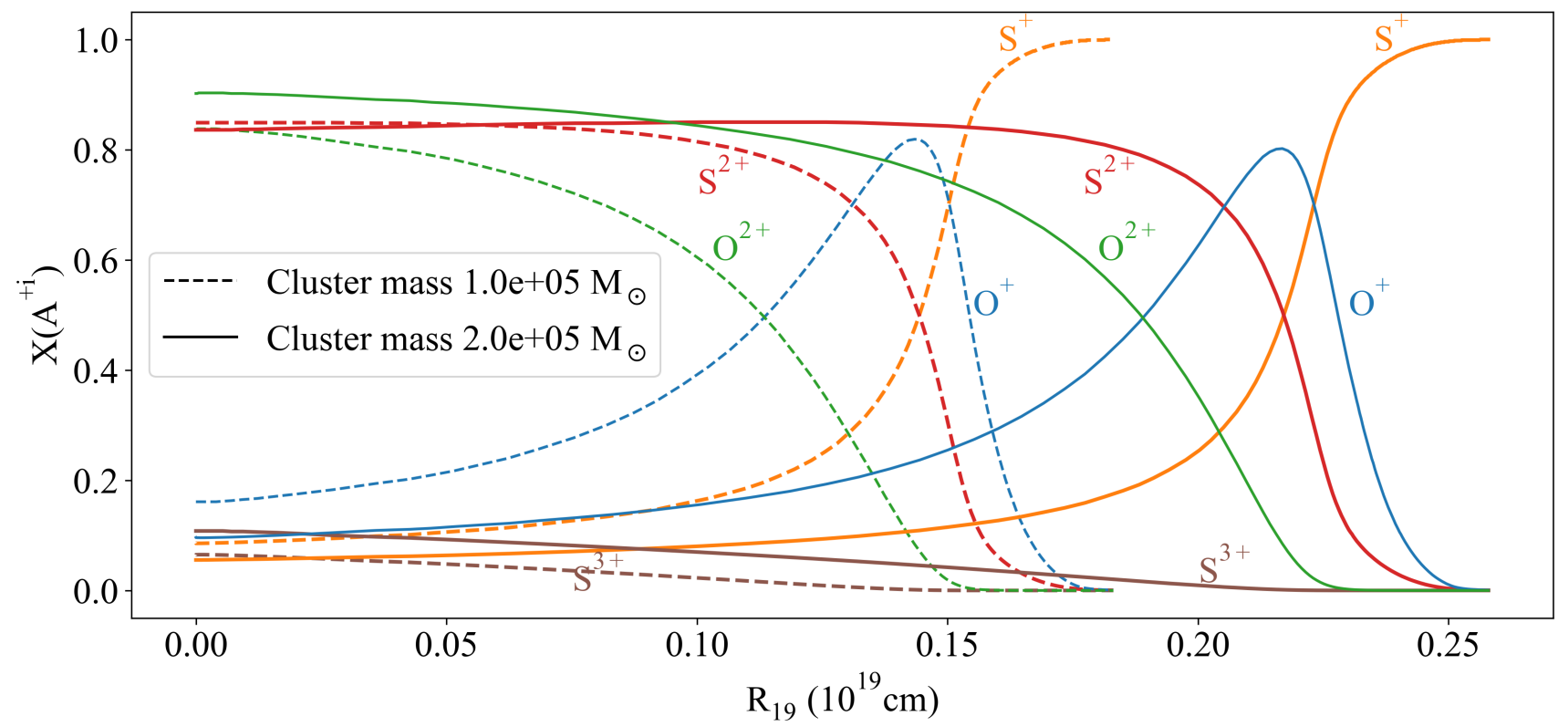

Figure 4. Ionization structure for sulphur and oxygen in two of the PopStar evolutionary synthesis models described in the text: $\log ($ age $)=5.48, Z_{\text {gas }}=0.004, \log \left(Z_{\star}\right)=-2.1$ and constant $n_{e}=100 \mathrm{~cm}^{3}$. Dashed lines represent a cluster mass of $10^{5} M_{\odot}$, solid lines correspond to $2 \cdot 10^{5} M_{\odot}$.

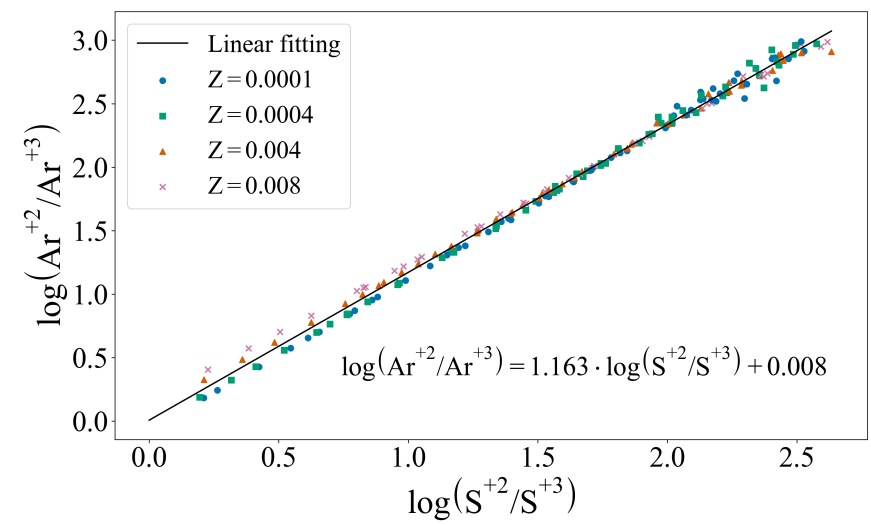

Figure 5. Relation between the ratio of twice to thrice ionized argon and sulphur abundances for the PopStar models grid described in the text.

nation contribution to the lines. To account for this feature, we used the correction provided by Liu et al. (2000):

$\frac{I([O I I] 7319 \AA+[O I I] 7330 \AA)}{I(H \beta)}=9.36 \cdot t^{0.44} \times \frac{O^{2+}}{H^{+}}$

Therefore:

$$
\begin{aligned}
T_{\text {high }}=T_{e}[O I I I] & =f\left(\frac{I([O I I I] 4363 \AA)}{I([S O I I I] 4959 \AA+[O I I I] 5007 \AA)}\right) \\
\frac{O^{+}}{H^{+}} & =f\left(\sum_{i} I_{i}([O I I]), T_{\text {low }}, n_{e}[S I I]\right) \\
\frac{O^{2+}}{H^{+}} & =f\left(\sum_{i} I_{i}([O I I I]), T_{\text {high }}, n_{e}[S I I]\right)
\end{aligned}
$$

and the total oxygen abundance is calculated as:

$$
\frac{O}{H}=\left(\frac{O^{+}}{H^{+}}+\frac{O^{2+}}{H^{+}}\right)
$$

The values for the ionic and total $\mathrm{O}$ abundance are given in Tables 6 and 7 respectively.

\subsubsection{Nitrogen:}

In the optical wavelength range, the nitrogen abundance is normaly derived from the $[N I I] \lambda \lambda 6548 \AA$, $6583 \AA$ lines. Our spectral resolution is enough to discriminate both lines from $H \alpha$. Nevertheless, as explained in Sec. 3.4, some objects required a fitting of four Gaussians to remove the contribution from a broad $H \alpha$ component.

A strong linear relation appears to exist between the $T_{e}[N I I]$ and $T_{e}[S I I I]$ values (Croxall et al. 2015) which validates the practice of adopting $T_{e}([N I I]) \approx T_{e}([S I I I])$ (Pérez-Montero \& Díaz 2003). For a given atomic data set, the $N^{+}$abundance is defined as:

$\frac{N^{+}}{H^{+}}=f\left(\sum_{i} I_{i}([N I I]), T_{l o w}, n_{e}[S I I]\right)$

Using the standard approach by Peimbert \& Costero (1969) which states that $N I / O I \approx N I I / O I I$, the nitrogen abundance can be calculated as:

$$
\begin{aligned}
\frac{N}{H} & =\frac{N}{O} \cdot \frac{O}{H} \\
& =\frac{N I I / H I I}{O I I / H I I} \cdot \frac{O}{H}
\end{aligned}
$$

Our derived ionic and total nitrogen abundances are given in Tables 6 and 7 respectively. 


\section{RESULTS AND DISCUSSION}

Table 2 includes emission lines data for two galaxies observed using ISIS-I and ISIS-II set ups respectively ${ }^{3}$. The table lists the equivalent width, the observed flux and the reddening-corrected intensity (where $H \beta=1000$ ). These entries include the correction from the underlying stellar continuum fitting.

For ${ }^{4} \mathrm{He}$ to be considered a "baryometer" 4 its primordial abundance $\left(Y_{P}\right)$ needs to be determined with high accuracy taking into account the many possible error sources (e.g. Davidson \& Kinman 1985) in particular the systematic errors (e.g. Peimbert et al. 2007b). The uncertainties can be separated into 5 groups: data acquisition and reduction, quantifying the spectra components, ionic abundances calculation procedures, ionization correction factors and total abundances determination, and finally, errors associated with the extrapolation to zero metals.

In what follows we will use our data to derive $Y_{P}$ as the extrapolation to zero metals of the relation between He and metal abundances, using as metallicity indicator sulphur and compare the results to those obtained using the classical approach of oxygen and nitrogen as metallicity indicators. We will also discuss the impact on the $Y_{P}$ value of the different sources of error.

\subsection{Data and reduction uncertainties}

Observational data from the near-infrared (NIR) is essential for accurate sulphur abundance determination. The [SIII] $\lambda \lambda 9069,9532 \AA$ lines are prominent features in this wavelength range. We have discussed in section 2 the difficulties that arise from observing in this region and how to circumvent them.

Since our current reduction pipeline does propagate the pixel flux uncertainty, theoretical ionic emissivity ratios may be compared with the observed ionic flux ratios as a qualitative check. The first and second column in Table 4 show the observed ratios $F([O I I I] \lambda 5007 \AA) / F(\lambda 4959 \AA)$ and $F([S I I I] \lambda 9532 \AA) / F(\lambda 9069 \AA)$ for our sample. The magnitudes are colour coded green, orange and red to represent deviations from the theoretical value by below $5 \%$, below $10 \%$ and above $10 \%$ respectively. A very good agreement for the oxygen ratio in the blue spectrograph arm can be noticed. Regarding the sulphur ratio in the red arm, a close to theoretical ratio is obtained when telluric corrections could be performed.

These results suggest a flux calibration within $5 \%$ which is the error assumed for the standard stars flux calibration curves.

\subsection{Flux uncertainty}

Flux uncertainties can have an impact on the physical parameters, e.g. a $10 \%$ uncertainty yields a temperature difference in $T$ [SIII] of around $500 \mathrm{~K}$. This translates into an abundance uncertainty of around 0.15 dex (see Kennicutt et al. 2003; Hägele et al. 2008; Dors et al. 2016). Fortunately, the random nature of this error minimizes its effect on the linear regression $Y_{P}$ vs. $\mathrm{S} / \mathrm{H}$ as long as its magnitude remains small.

\subsection{Nebular continuum uncertainty}

Our sample selection criterion of $E W(H \alpha)>200 \AA$ guarantees an ionizing cluster younger than $10 \mathrm{Myr}$ (see Dottori \& Bica 1981;

\footnotetext{
3 The complete table for all the objects is published in electronic form

4 This term, coined by David Schramm, reflects the dependence of primordial light element abundances on the cosmic density of baryons and the baryon to photon ratio: $\eta$
}

Leitherer et al. 1999). The youngest burst in our sample would be that of $S H O C 220$ with $E W(H \alpha)=1310 \AA$. In such young bursts, the luminosity is dominated by the emission. However, to guarantee the most accurate abundance derivation from the weak helium lines, the underlying stellar absorption needs to be quantified for which previous subtraction of the nebular continuum is required.

The nebular continuum can be calculated from first principles, as described in section 3.2. This calculation, however, requires the input of some characteristic electron temperature and density, as well as flux. These parameters can be inferred from the data when it includes the Balmer and/or Paschen jumps. In the former case, the continuum temperature $\left(T_{B a c}\right)$ can be related to the jump flux intensity $(B J)$ and the $H_{11} \lambda 3770 \AA$ Balmer line, following Liu et al. (2001):

$T_{\text {Bac }}=368 \cdot\left(1+0.259 \cdot y^{+}+3.409 \cdot y^{2+}\right)\left(\frac{B J}{H_{11}}\right)$

where the Balmer jump flux is calculated assuming linear continuum regions before and after the discontinuity. In this work (see 3.2) the nebular continuum intensity was calibrated using a Zanstra-like approach, eq. 15, using a hydrogen recombination line. We used $\boldsymbol{H} \boldsymbol{\alpha}$ due to its relatively low contamination by stellar absorption. The de-reddened spectrum of SHOC579 is shown in figure 6 , where we can distinguish the small Balmer jump and the $H_{20} \lambda 3683 \AA$ line. Using eq. $29, T_{B a c}=13200 \pm 3000 \mathrm{~K}$ in agreement with the values estimated from the oxygen and sulfur emission line diagnostics, although this measurement has a larger uncertainty. This was also the conclusion reached by García-Rojas et al. (2012) for planetary nebulae: The large number of emission lines in the proximity of the hydrogen discontinuities leaves very few points to perform the linear fits. The situation is worse for the Paschen jump due to the telluric contamination on the continuum. An optimum solution would consist of fitting simultaneously the nebular and stellar continua to the observed spectrum. This approach has been applied successfully by Gomes \& Papaderos (2017) in their FADO package. Fortunately, for the electron temperatures and densities commonly encountered in HII galaxies, the featureless nebular continuum is almost independent of these parameters (Zhang et al. 2004). Consequently, for this region, the nebular continuum shape can be obtained assuming $T_{e}=10000 \mathrm{~K}$ and $n_{e}=100.0$. This is also the approach employed by FADO for input spectra which don't include the hydrogen discontinuities.

\subsection{Uncertainties in the underlying population subtraction}

Rosales-Ortega (2006) illustrated the impact of including a spectral synthesis analysis on the helium abundance calculation. The absorption on the recombination lines can be checked in Fig. 6, which displays the fitted stellar continuum for SHOC579 using STARLIGHT. Our SSP library is particularly tailored for young and low metallicity populations (as described in section 3.3). In these galaxies, as it can be seen on the figure, the absorption features may be absent on the observed spectrum. Consequently, it is challenging for spectra synthesis codes to fit a stellar continuum. Within our SSPs library wavelength coverage, our spectra do not include any significant absorption features. To avoid overestimations on the stellar velocity dispersion, our fittings are constrained to a maximum calculated from the mean velocity dispersion of the CaII triplet lines. If these were not observed, the $[O I I I] \lambda 5007 \AA$ velocity dispersion was adopted $\left(20<\sigma_{[O I I I] \lambda 5007 \AA}<80 \mathrm{~km} / \mathrm{s}\right)$.

The histograms in Fig. 7 display the stellar population output from the STARLIGHT fit to SHOC579. The left and right panels correspond to the mass and light weighted population distribution respectively versus the age bin. The metallicities are colour coded in the $0.00037<Z<0.03153$ interval. The implications from these results are as expected: the older populations dominate the mass, while the younger one dominates the light. A 


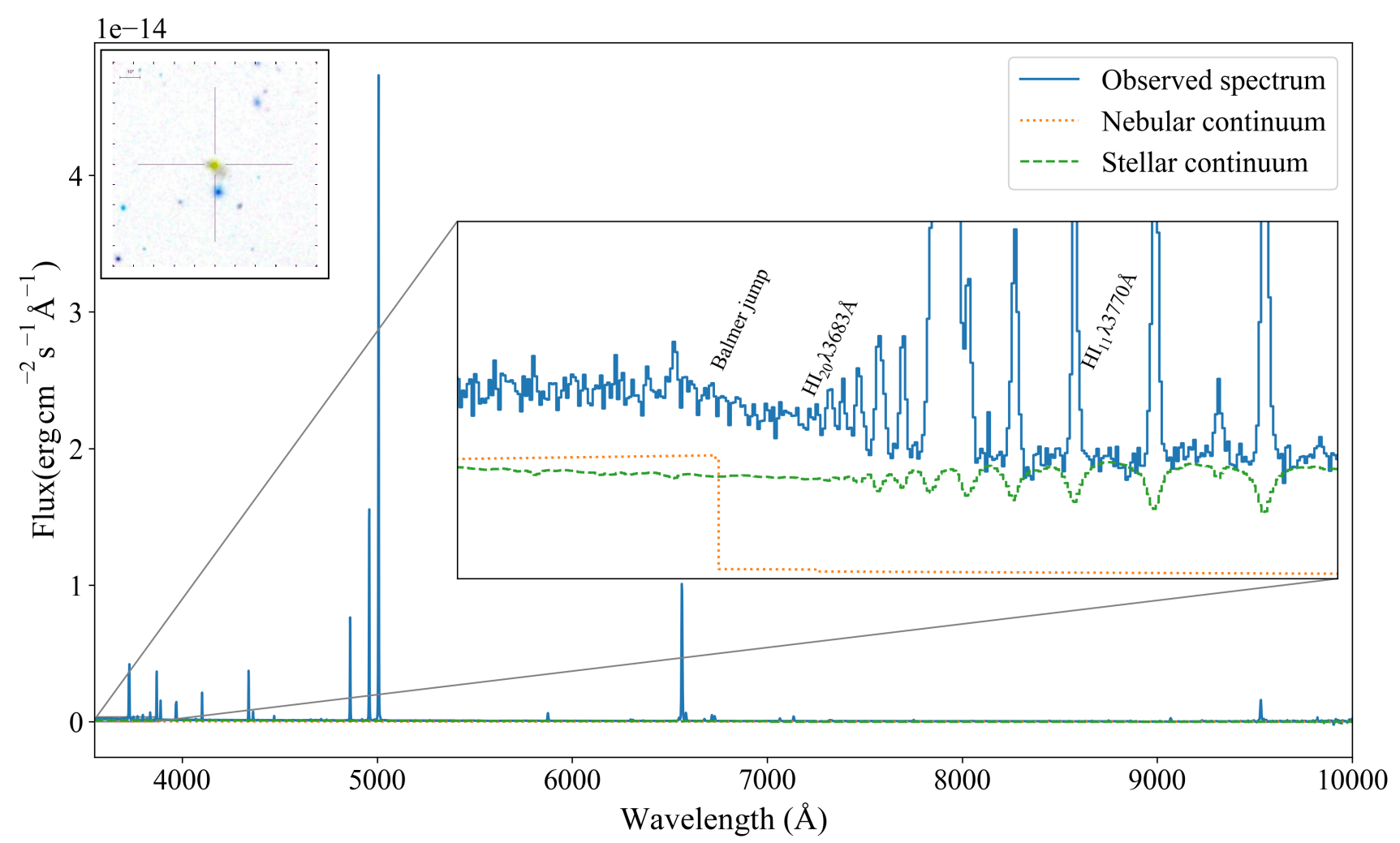

Figure 6. Spectral components for SHOC579. The object image belongs to the SDSS database.
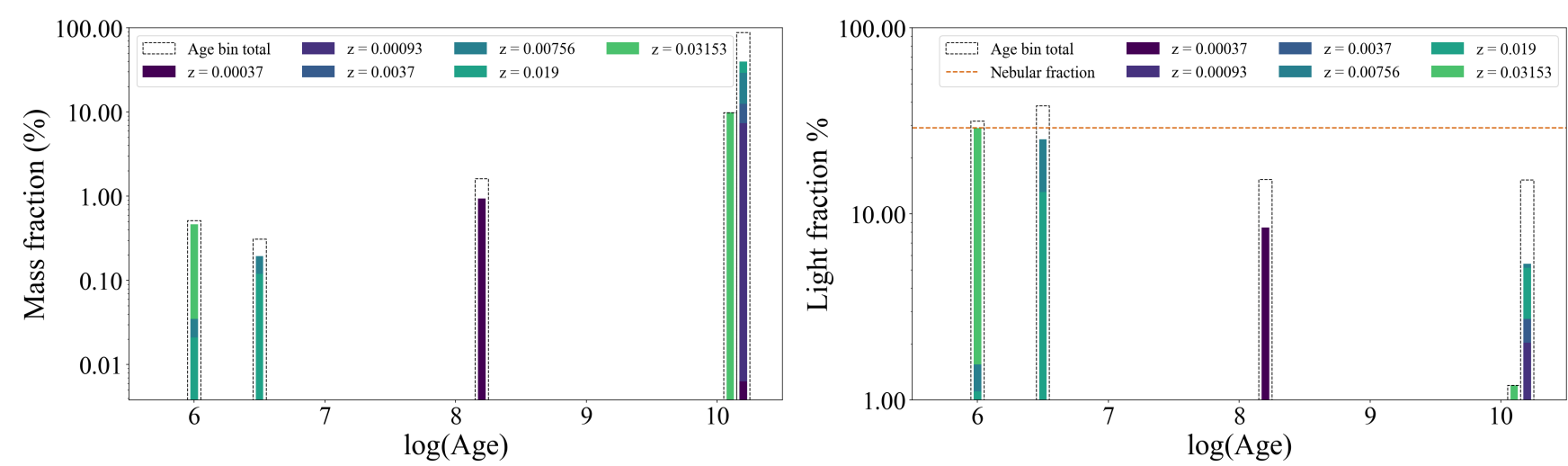

Figure 7. Mass (left) and light (right) weighted population histograms for SHOC579 from STARLIGHT. The bar colours represent the stellar population metallicity, while the dotted line represents the total contribution from several metallicities. The dotted horizontal line represents the light from the nebular continuum as a percentage of the total stellar continuum.

metallicity gradient may be noted with older stars displaying a lower metal content. Additional information which can be derived from this analysis includes the current stellar mass. For SHOC579 $\log \left(M_{T}\right)=8.05$ which implies that the host galaxy should be relatively evolved, although we cannot say anything from the observed spectrum. Finally, to compare the nebular and stellar continuum luminosity, we integrate the total flux from each continuum for our wavelength range. This is displayed on the right hand side histogram in Fig. 7 as a dashed horizontal line that quantifies the nebular-to-stellar luminosity percentage. For SHOC579, the nebular luminosity is almost $20 \%$ of the stellar luminosity and is of the same order as the brightest population. This illustrates the impact of the gas luminosity whenever we seek to quantify the youngest populations on bursts of star formation.

While the input spectra on these STARLIGHT fittings have been corrected from reddening, the program also computes a uniform extinction of the stellar populations. For most objects this extinction is very low or zero suggesting that the extinction in the ionized gas is similar to that of the immersed stellar population. However, this is not the case for SHOC579 for which $A_{V ; \text { stellar }}=0.43$, while the extinction predicted from the Balmer decrement is $A_{V ; \text { gas }}=0.061 \pm 0.002$ assuming a Gordon et al. (2003) extinction law with $R_{V}=3.4$. The impact on the reddening correction is further discussed in the next section.

The goodness of the STARLIGHT fitting depends largely on 
the available amount of unmasked spectrum and on the intensity of the absorption features. Configuration II covers almost $1000 \AA$ more than Configuration I. In the latter case, the unmasked continuum is practically featureless: no sizable absorptions are detected even on the helium lines. In these cases, the SSP synthesis result consists in just a single young stellar population. Quantifying the accuracy and precision of these fits remains a challenge for a SSP algorithm (Cid Fernandes et al. 2013). Still, even with a poor stellar population fit, the current analysis should not be less accurate than results using other methods for quantifying the underlying stellar population in previous $Y_{P}$ determinations. We have already mentioned the approach by Rosales-Ortega (2006) followed by Peimbert et al. (2007b) who computed the helium abundance taking $E W_{a b} \approx 2 \AA$ from González-Delgado et al. (2005) models. In the multidimensional analysis by Aver et al. (2015), they consider two constant values for the underlying absorption in each object: one for the hydrogen lines and another one for the helium lines. Finally, Izotov et al. (2014), uses for all the HII regions an underlying absorption for each helium line from the models of González-Delgado et al. (2005) and the Starburst99 high resolution spectra (Leitherer et al. 1999).

The solution towards a better stellar continuum fit relies on increasing the wavelength coverage while preserving the temporal and spectral resolution. Currently, the simple stellar libraries by (Bruzual \& Charlot 2003), which reach $9500 \AA$, are being updated with younger stellar populations ${ }^{5}$. Consequently, it will be possible to fit the CaII triplet ( $\lambda 8498 \AA, 8542 \AA, 8662 \AA)$ in observations such as these ones. This will provide an anchor to the older stellar population.

\section{5 $\mathrm{T}_{e}, \mathrm{n}_{e}$ and ionic abundances}

At this point the emission line fluxes can be used to calculate $T_{e}$ and $n_{e}$. The extinction still needs to be calculated, though. A canonical approach consists in comparing the theoretical ratio of the Balmer lines with the observed flux ratios along with a reddening law. In HII regions, however, there is some degeneracy in this approach since the observed recombination lines could also be affected by underlying stellar absorption. The solution we chose is to apply the following scheme:

(i) An initial reddening correction is obtained from the Balmer decrement assuming $T_{e}=10000 \mathrm{~K}$ and $n_{e}=100 \mathrm{~cm}^{-3}$; the theoretical ratios are calculated using PyNeb. We use Gordon et al. (2003) reddening law as appropriate for young starforming bursts with $R_{V}=3.4$.

(ii) The ionic abundances are calculated.

(iii) The nebular contribution is calculated using the physical parameters determined from the previous step and the dereddened $H \alpha$ emission.

(iv) The nebular contribution is subtracted from the dereddened object spectrum and the stellar continuum is fitted.

(v) The nebular and stellar continua are removed from the spectrum and the corrected emission lines are measured.

(vi) A new reddening coefficient is calculated. The previous steps are repeated using the measurements from this iteration as initial values.

(vii) From the second iteration we obtain the final line intensities and from them the element abundances as discussed in section 3.5 , which we will use in a regression to estimate $Y_{\boldsymbol{P}}$ as we will discuss in the following section.

Since both the extinction and underlying stellar absorption to the

5 Soon to be available, Aida Wofford and Gustavo Bruzual, private communication. recombination lines increase towards shorter wavelengths the reddening coefficient decreases in the second iteration. In one object, it decreased to almost a half of its original value. However, since most of these objects show low extinction this change did not produce a great impact on the ionic abundances. As mentioned in the previous section, the stellar bases employed on the stellar population synthesis did not reach beyond $7000 \AA$. Consequently, none of the Paschen lines could be included on the reddening calculation as they were not corrected from stellar absorption. Still, even if this would had been the case, the Paschen lines within our wavelength range $\left(P_{20}\right.$ to $\left.P_{9}\right)$ have a low signal to noise ratio due to the telluric contamination. Consequently, their weight on the $c(H \boldsymbol{\beta})$ linear fit is very low compared to the one from the Balmer $H \alpha$, $H \beta, H \gamma$ and $H \delta$ lines. As an additional precaution, to avoid flux calibration mismatch between the spectrograph arms, the lines in the blue arm were normalized with respect to $H \beta$, while for those in the red arm we used the most intense hydrogen recombination line available depending on the configuration: $H \alpha, P_{8}$ or $P_{9}$. Afterwards, these lines were multiplied by the theoretical emissivity ratio $\left(\epsilon_{\lambda, \text { red arm }} / \epsilon_{H \beta}\right)$ so that the lines in both arms could be compared.

A direct abundance estimate, such as the one described in section 3.5, requires measurement of both temperature and density sensitive line ratios. For our data we can determine $T_{e}[O I I I]$ and $T_{e}[S I I I]$ for both configurations. These values are given in Table $4 . T_{e}[O I I I]$ has been exclusively used in the helium abundance determination. This is due to the characteristics of the objects involved: low metallicity and high ionization factors. This guarantees strong lines, hence good precision. In contrast the $T_{e}[S I I I]$ can be measured in ionized gas with metallicity up to solar. This is an important asset in order to determine better the slope of the relation and therefore to extend the linear regression for determining $Y_{P}$ (see the following subsection). On the other hand, stronger lines imply smaller uncertainty for the gradient (Berg et al. 2015, CHAOS survey). Berg and collaborators use $T[S I I I], T[N I I]$ and the theoretical relation by Garnett (1992) to estimate the high ionization $T_{e}[O I I I]$. This was shown in Fig. 3. The orthogonal linear regression from our data agrees with the estimates in the literature. The dotted line represents the estimate from Garnett (1992) while the dashed lines are the photo-ionization models by Pérez-Montero \& Díaz (2005) and Pérez-Montero (2014). The different gradients in these two linear models can be explained by the different atomic data sources for the $S^{2+}$ collisional strengths. It should be noticed that all lines overlap in the $10,000-15,000 \mathrm{~K}$ region. This is also the region, where $T_{e}[S I I I] \backsim T_{e}[O I I I]$ and where most of our data falls. The well known extremely low metallicity HII galaxy IZw18 lies in the outskirts of this relation displaying a higher than expected $T_{e}[S I I I]$. Its chemical analysis, however, is still under debate. In a recent paper by Kehrig et al. (2016), this galaxy was analyzed using integral field spectroscopy to compare the emission from both NW and SE galaxy knots. The NW region is larger, has a higher electron temperature, lower metallicity and a stronger continuum. In contrast, the smaller SE region has slightly higher metallicity and lower electron temperature. Their estimates: $T_{e}[O I I I]_{N W}=$ $23000 \pm 700 \mathrm{~K}$ and $T_{e}[O I I I]_{S W}=19600 \pm 600 \mathrm{~K}$ agree within errors with our values shown in Table 4 . Given the linear relation between both temperatures for our sample objects, as discussed in section 3.5, we adopted the following compromise: the most precise temperature (either $T_{e}[O I I I]$ or $T_{e}[S I I I]$ ) is used to characterize the galaxy. For example, if a low redshift object has a large error for the $[O I I I] \lambda 4363 \AA$ line because it lies very close to the spectrum edge, the high ionization temperature will be calculated using $T_{e}[S I I I]$ in eq. 16 . This will minimize the dispersion on the regression hence improving the extrapolation for determining $Y_{P}$, as will be discussed in the following sections.

The abundances, as described in section 3.5, are determined using the canonical direct method except for two points. 
Table 4. Electron temperature and density. A superscript on the object name represents the element used for the temperature. In these cases the missing low or high ionization temperature was derived from eq. 16. The colour coding in columns (2) and (3) represents the departure from the theoretical ratio: green below $5 \%$, yellow below $10 \%$ and red above $10 \%$

\begin{tabular}{|c|c|c|c|c|c|}
\hline HII Galaxy & $\frac{[O \text { II I I }] \lambda 5007 \AA}{[O I I I] \lambda 4959 \AA}$ & $\frac{[S I I I] \lambda 9531 \AA}{[S I I I] \lambda}$ & $n_{e}[S I I]\left(\mathrm{cm}^{-3}\right)$ & $T_{e}[S I I I](K)$ & $T_{e}[O I I I](K)$ \\
\hline FTDTR-1 $^{S}$ & $2.99 \pm 0.065$ & $2.39 \pm 0.037$ & $49.9 \pm 21$ & $14700 \pm 710$ & $17900 \pm 910$ \\
\hline $\mathrm{IZw} 18^{\mathrm{S}}$ & $3.08 \pm 0.079$ & $2.56 \pm 0.067$ & $49.7 \pm 22$ & $21700 \pm 1800$ & $18300 \pm 900$ \\
\hline MRK36-A1 & $3.01 \pm 0.078$ & $2.39 \pm 0.020$ & $50.0 \pm 22$ & $15100 \pm 670$ & $14900 \pm 850$ \\
\hline MRK36-A2 & $3.03 \pm 0.096$ & $2.59 \pm 0.014$ & $88.3 \pm 31$ & $12900 \pm 290$ & $15800 \pm 430$ \\
\hline MRK475 & $3.05 \pm 0.091$ & $2.70 \pm 0.013$ & $50.7 \pm 21$ & $13400 \pm 340$ & $14300 \pm 410$ \\
\hline FTDTR-2 $O$ & $3.11 \pm 0.083$ & $2.36 \pm 0.058$ & $50.3 \pm 22$ & $14100 \pm 1700$ & $13900 \pm 760$ \\
\hline $\mathrm{IZw} 70^{S}$ & $2.99 \pm 0.089$ & $2.54 \pm 0.018$ & $50.8 \pm 22$ & $10800 \pm 450$ & $12900 \pm 710$ \\
\hline MRK689 $9^{S}$ & $3.04 \pm 0.077$ & $2.45 \pm 0.015$ & $51.5 \pm 22$ & $10200 \pm 360$ & $11100 \pm 850$ \\
\hline MRK67 ${ }^{S}$ & $3.05 \pm 0.097$ & $2.51 \pm 0.019$ & $81.5 \pm 38$ & $12600 \pm 450$ & $14800 \pm 610$ \\
\hline FTDTR-3 & $3.03 \pm 0.071$ & $2.37 \pm 0.011$ & $49.2 \pm 21$ & $12800 \pm 420$ & $12900 \pm 360$ \\
\hline SHOC022 & $3.11 \pm 0.034$ & $2.42 \pm 0.020$ & $49.5 \pm 22$ & $11400 \pm 580$ & $12200 \pm 620$ \\
\hline FTDTR-4 & $3.09 \pm 0.065$ & $2.41 \pm 0.018$ & $49.0 \pm 22$ & $12200 \pm 580$ & $15000 \pm 330$ \\
\hline $\mathrm{SHOC} 220^{\circ}$ & $2.88 \pm 0.0021$ & $2.36 \pm 0.059$ & $128 \pm 81$ & $17500 \pm 1200$ & $19500 \pm 400$ \\
\hline FTDTR-5 ${ }^{O}$ & $3.04 \pm 0.065$ & $2.47 \pm 0.073$ & $165 \pm 110$ & $14400 \pm 2200$ & $13100 \pm 1100$ \\
\hline FTDTR-6 & $3.04 \pm 0.069$ & $2.60 \pm 0.0086$ & $49.9 \pm 22$ & $12100 \pm 440$ & $12800 \pm 400$ \\
\hline FTDTR-7 & $3.05 \pm 0.057$ & $2.50 \pm 0.0085$ & $132 \pm 20$ & $13300 \pm 300$ & $13800 \pm 200$ \\
\hline MRK $627^{S}$ & $3.04 \pm 0.056$ & $2.49 \pm 0.019$ & $50.6 \pm 22$ & $10300 \pm 460$ & $11300 \pm 770$ \\
\hline $\mathrm{SHOC} 592^{O}$ & $3.02 \pm 0.0066$ & $2.60 \pm 0.13$ & $149 \pm 30$ & $9740 \pm 750$ & $10400 \pm 300$ \\
\hline PHL293B & $3.08 \pm 0.076$ & $2.65 \pm 0.080$ & $112 \pm 31$ & $14000 \pm 500$ & $15800 \pm 230$ \\
\hline SHOC588 & $3.15 \pm 0.044$ & $2.54 \pm 0.069$ & $50.3 \pm 22$ & $11900 \pm 390$ & $10500 \pm 190$ \\
\hline $\mathrm{SHOC}_{036} \mathrm{O}$ & $3.04 \pm 0.013$ & - & $49.9 \pm 21$ & - & $16300 \pm 550$ \\
\hline $\mathrm{SHOC} 575^{\mathrm{O}}$ & $2.96 \pm 0.022$ & $1.90 \pm 0.033$ & $125 \pm 43$ & $11700 \pm 580$ & $10700 \pm 340$ \\
\hline SHOC579 & $3.05 \pm 0.0031$ & $5.80 \pm 0.15$ & $147 \pm 8.0$ & $11600 \pm 160$ & $12400 \pm 50$ \\
\hline FTDTR-8 ${ }^{O}$ & $3.00 \pm 0.0060$ & $2.96 \pm 0.078$ & $50.2 \pm 22$ & $13400 \pm 2000$ & $15100 \pm 550$ \\
\hline $\mathrm{SHOC} 263^{\circ}$ & $2.98 \pm 0.0097$ & - & $227 \pm 83$ & - & $11000 \pm 400$ \\
\hline FTDTR-9 & $3.09 \pm 0.076$ & $2.67 \pm 0.069$ & $147 \pm 62$ & $11400 \pm 890$ & $11600 \pm 780$ \\
\hline FTDTR-10 ${ }^{O}$ & $2.88 \pm 0.0061$ & $2.73 \pm 0.12$ & $666 \pm 200$ & - & $14200 \pm 720$ \\
\hline
\end{tabular}

First, the $\mathrm{O}^{+} / \mathrm{H}^{+}$determination for many objects depends on the $[O I I] \lambda \lambda 7319 \AA, 7330 \AA$ lines as the stronger [OII] $\lambda \lambda 3726 \AA, 3729 \AA$ doublet is not observed with Configuration I. These weaker lines are affected by a recombination effect and in some cases also by sky emission. These effects are treated using the recombination prediction from eq. 24 and the telluric correction respectively. The possible underlying stellar absorption can not be properly accounted for as those lines are beyond the wavelength limit of the used stellar database, as already discussed. It is expected, however, that this error should be very small given the young age, by selection, of the sample.

The second difference is the calculation of the $\operatorname{ICF}\left(\mathrm{S}^{3+}\right)$ using argon (see section 3.5.2 and Fig. 5). Even when $S^{+3}$ is expected to be present in these objects on account of their high excitation, still it is hard to quantify. Even though data exists for $[S I V] 10.51 \mu \mathrm{m}$ or $[S I I I] 18.71 \mu \mathrm{m}$ these lines have also a recombination component. It is essential to anchor the infrared results to optical observations by including measurements of a nearby recombination line. Fig. 8 compares the predictions using our ICFs with available infrared data for $[S I V] 10.51 \mu \mathrm{m}$ and $[S I I I] 18.71 \mu \mathrm{m}$ in the literature as compiled by Dors et al. (2016). While the empirical results are biased towards low temperatures both ICFs cover the same region. The uncertainty in these ICFs comes both from the error propagation on the ionic abundances, and from the fit using eq. 21 . The helium, oxygen and nitrogen ionic abundances for our sample are shown in Table 5, while Table 6 displays their measured sulphur and argon ionic abundances.

\section{6 $Y_{P}$ regressions}

After the pioneering Peimbert \& Torres-Peimbert (1974) paper, $Y_{P}$ has been calculated as the extrapolation to zero metals (oxy-

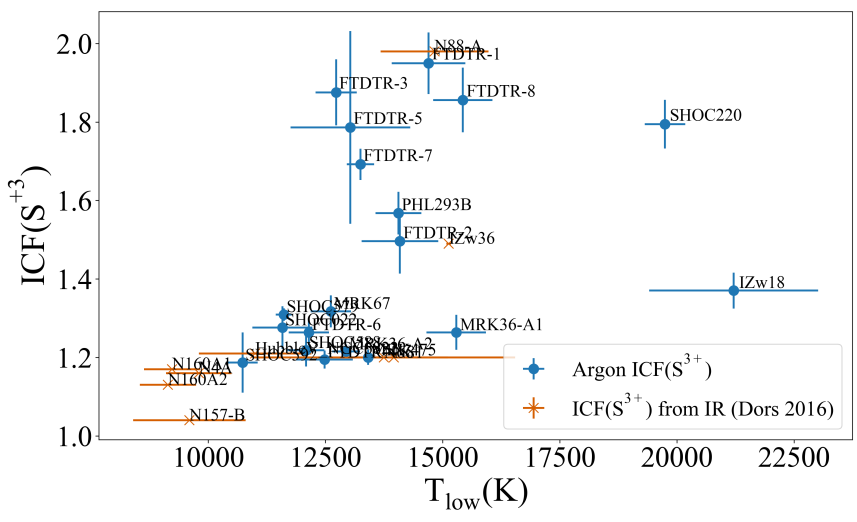

Figure 8. ICF $\left(S^{3+}\right)$ vs. the low ionization temperature. ICFs for our objects (blue points) were calculated from the Ar lines following the correlation in eq. 21. The red symbols are objects from Dors et al. (2016) with [SIII] and [SIV] infrared observations.

gen) of the He abundance by mass. Fig. 9 shows $\Delta Y v s \Delta Z$ using as metallicity indicator $(Z)$ oxygen, nitrogen or sulphur. The abundances are given in Table 7 where, as a superscript to the object name on Column (1), we indicate which objects were used for each $Y_{P}$ determination (using either $\mathrm{O}, \mathrm{N}$, or $\mathrm{S}$ as metal indicator). The helium abundance is given in column (2); the helium mass fractions, calculated using either oxygen or sulphur are columns (3) and (4). Columns (4), (5) and (6) show the oxygen, nitrogen and sulphur abundances respectively.

The $\Delta Y v s \Delta Z$ gradient has been explained by chemical evolution models (e.g. Carigi \& Peimbert 2010). A strong Y-N cor- 
Table 5. Helium, oxygen and nitrogen ionic abundances.

\begin{tabular}{lccccc}
\hline HII Galaxy & $\mathrm{He}^{+} / \mathrm{H}^{+}$ & $\mathrm{He}^{2+} / \mathrm{H}^{+}$ & $12+\log \left(\mathrm{O}^{+} / \mathrm{H}^{+}\right)$ & $12+\log \left(\mathrm{O}^{2+} / \mathrm{H}^{+}\right)$ & $12+\log \left(\mathrm{N}^{+} / \mathrm{H}^{+}\right)$ \\
\hline FTDTR-1 & $0.0977 \pm 0.0011$ & $0.0012 \pm 0.0002$ & $7.29 \pm 0.10$ & $7.75 \pm 0.053$ & $5.38 \pm 0.050$ \\
IZw18 & $0.0768 \pm 0.0028$ & $0.00053 \pm 0.0$ & $6.48 \pm 0.12$ & $6.95 \pm 0.069$ & $4.74 \pm 0.062$ \\
MRK36-A1 & $0.0795 \pm 0.0082$ & $0.0018 \pm 0.0002$ & $7.32 \pm 0.091$ & $7.73 \pm 0.071$ & $5.56 \pm 0.21$ \\
MRK36-A2 & $0.0801 \pm 0.0097$ & $0.0012 \pm 0.0$ & $7.68 \pm 0.051$ & $7.68 \pm 0.038$ & $5.67 \pm 0.33$ \\
MRK475 & $0.0883 \pm 0.0045$ & $0.0017 \pm 0.0001$ & $7.50 \pm 0.059$ & $7.83 \pm 0.043$ & $5.75 \pm 0.25$ \\
FTDTR-2 & $0.0756 \pm 0.0033$ & $0.0010 \pm 0.0002$ & - & $7.88 \pm 0.069$ & $5.53 \pm 0.067$ \\
IZw70 & $0.0943 \pm 0.0032$ & $0.00058 \pm 0.0001$ & $8.15 \pm 0.11$ & $7.99 \pm 0.055$ & $6.40 \pm 0.045$ \\
MRK689 & $0.0775 \pm 0.0068$ & - & $8.35 \pm 0.095$ & $8.19 \pm 0.049$ & $6.31 \pm 0.041$ \\
MRK67 & $0.0910 \pm 0.012$ & $0.00096 \pm 0.0$ & $7.79 \pm 0.084$ & $8.00 \pm 0.047$ & $5.88 \pm 0.19$ \\
FTDTR-3 & $0.0847 \pm 0.0030$ & - & $7.47 \pm 0.074$ & $8.12 \pm 0.043$ & $5.61 \pm 0.24$ \\
SHOC022 & $0.0838 \pm 0.0018$ & $0.0014 \pm 0.0001$ & $7.97 \pm 0.13$ & $7.97 \pm 0.069$ & $6.24 \pm 0.055$ \\
FTDTR-4 & $0.0878 \pm 0.0015$ & $0.00075 \pm 0.0$ & $7.87 \pm 0.11$ & $7.85 \pm 0.028$ & $6.04 \pm 0.049$ \\
SHOC220 & $0.0989 \pm 0.0023$ & $0.0018 \pm 0.0001$ & $6.80 \pm 0.043$ & $7.52 \pm 0.024$ & $5.56 \pm 0.075$ \\
FTDTR-5 & $0.0779 \pm 0.0042$ & - & - & $8.06 \pm 0.11$ & - \\
FTDTR-6 & $0.0869 \pm 0.0019$ & $0.00088 \pm 0.0$ & $7.80 \pm 0.090$ & $8.00 \pm 0.045$ & $5.84 \pm 0.16$ \\
FTDTR-7 & $0.0864 \pm 0.0013$ & $0.00041 \pm 0.0$ & $7.49 \pm 0.050$ & $8.05 \pm 0.020$ & $5.72 \pm 0.022$ \\
MRK627 & $0.0845 \pm 0.0040$ & - & $8.30 \pm 0.12$ & $8.12 \pm 0.060$ & $6.54 \pm 0.051$ \\
SHOC592 & $0.0962 \pm 0.0080$ & - & $7.74 \pm 0.061$ & $8.18 \pm 0.043$ & $6.59 \pm 0.037$ \\
PHL293B & $0.0766 \pm 0.0024$ & $0.0017 \pm 0.0002$ & $6.73 \pm 0.052$ & $7.70 \pm 0.021$ & - \\
SHOC588 & $0.0940 \pm 0.0084$ & $0.00059 \pm 0.0$ & $7.38 \pm 0.055$ & $8.18 \pm 0.028$ & $6.25 \pm 0.034$ \\
SHOC036 & $0.102 \pm 0.010$ & $0.0028 \pm 0.0007$ & $6.75 \pm 0.046$ & $7.69 \pm 0.038$ & $5.57 \pm 0.051$ \\
SHOC575 & $0.0977 \pm 0.0018$ & - & $7.57 \pm 0.063$ & $8.18 \pm 0.046$ & $6.66 \pm 0.038$ \\
SHOC579 & $0.0991 \pm 0.0049$ & $0.00040 \pm 0.0$ & $7.17 \pm 0.023$ & $8.05 \pm 0.0057$ & $6.35 \pm 0.014$ \\
FTDTR-8 & $0.0750 \pm 0.0018$ & - & $7.12 \pm 0.11$ & $7.86 \pm 0.045$ & $5.27 \pm 0.096$ \\
SHOC263 & $0.0866 \pm 0.0056$ & - & $8.19 \pm 0.11$ & $8.04 \pm 0.057$ & $6.63 \pm 0.053$ \\
FTDTR-9 & $0.0890 \pm 0.0032$ & $0.0016 \pm 0.0001$ & $8.00 \pm 0.23$ & $8.05 \pm 0.095$ & $6.27 \pm 0.086$ \\
FTDTR-10 & $0.0783 \pm 0.0026$ & $0.0010 \pm 0.0002$ & $7.22 \pm 0.13$ & $7.79 \pm 0.071$ & $5.79 \pm 0.21$ \\
\hline & & & & &
\end{tabular}

Table 6. Sulphur and argon ionic abundances.

\begin{tabular}{lccccc}
\hline HII Galaxy & $12+\log \left(\mathrm{S}^{+} / \mathrm{H}^{+}\right)$ & $12+\log \left(\mathrm{S}^{2+} / \mathrm{H}^{+}\right)$ & $I C F\left(\mathrm{~S}^{3+}\right)$ & $12+\log \left(\mathrm{Ar} \mathrm{r}^{2+} / \mathrm{H}^{+}\right)$ & $12+\log \left(\mathrm{Ar} \mathrm{r}^{3+} / \mathrm{H}^{+}\right)$ \\
\hline FTDTR-1 & $5.15 \pm 0.041$ & $5.83 \pm 0.036$ & $1.95 \pm 0.078$ & $5.16 \pm 0.043$ & $5.18 \pm 0.062$ \\
IZw18 & $4.62 \pm 0.050$ & $5.08 \pm 0.048$ & $1.37 \pm 0.046$ & $4.55 \pm 0.056$ & $4.16 \pm 0.090$ \\
MRK36-A1 & $5.31 \pm 0.038$ & $5.97 \pm 0.035$ & $1.26 \pm 0.045$ & $5.40 \pm 0.037$ & $4.78 \pm 0.080$ \\
MRK36-A2 & $5.43 \pm 0.024$ & $6.16 \pm 0.021$ & $1.22 \pm 0.018$ & $5.53 \pm 0.023$ & $4.79 \pm 0.037$ \\
MRK475 & $5.45 \pm 0.027$ & $6.23 \pm 0.024$ & $1.20 \pm 0.019$ & $5.64 \pm 0.026$ & $4.85 \pm 0.043$ \\
FTDTR-2 & $5.28 \pm 0.054$ & $5.99 \pm 0.046$ & $1.50 \pm 0.082$ & $5.38 \pm 0.058$ & $5.07 \pm 0.10$ \\
IZw70 & $5.91 \pm 0.043$ & $6.40 \pm 0.037$ & - & $5.80 \pm 0.042$ & - \\
MRK689 & $5.93 \pm 0.039$ & $6.56 \pm 0.033$ & - & $5.84 \pm 0.038$ & - \\
MRK67 & $5.54 \pm 0.037$ & $6.21 \pm 0.033$ & $1.32 \pm 0.041$ & $5.68 \pm 0.037$ & $5.15 \pm 0.056$ \\
FTDTR-3 & $5.23 \pm 0.032$ & $6.16 \pm 0.029$ & $1.88 \pm 0.084$ & $5.59 \pm 0.031$ & $5.53 \pm 0.040$ \\
SHOC022 & $5.77 \pm 0.053$ & $6.42 \pm 0.045$ & $1.28 \pm 0.054$ & $5.74 \pm 0.051$ & $5.14 \pm 0.087$ \\
FTDTR-4 & $5.64 \pm 0.046$ & $6.24 \pm 0.040$ & $1.19 \pm 0.023$ & $5.64 \pm 0.045$ & $4.87 \pm 0.038$ \\
SHOC220 & $4.73 \pm 0.024$ & $5.58 \pm 0.017$ & $1.79 \pm 0.062$ & $5.02 \pm 0.030$ & $4.93 \pm 0.028$ \\
FTDTR-5 & $5.30 \pm 0.090$ & $6.05 \pm 0.076$ & $1.79 \pm 0.25$ & $5.47 \pm 0.093$ & $5.37 \pm 0.13$ \\
FTDTR-6 & $5.48 \pm 0.037$ & $6.36 \pm 0.033$ & $1.26 \pm 0.032$ & $5.72 \pm 0.036$ & $5.06 \pm 0.050$ \\
FTDTR-7 & $5.27 \pm 0.020$ & $6.09 \pm 0.017$ & $1.69 \pm 0.040$ & $5.50 \pm 0.019$ & $5.34 \pm 0.020$ \\
MRK627 & $5.99 \pm 0.049$ & $6.55 \pm 0.041$ & - & $5.89 \pm 0.049$ & - \\
SHOC592 & $6.07 \pm 0.036$ & $6.69 \pm 0.031$ & $1.19 \pm 0.077$ & $5.89 \pm 0.036$ & $5.10 \pm 0.23$ \\
PHL293B & $5.12 \pm 0.031$ & $5.95 \pm 0.031$ & $1.57 \pm 0.054$ & $5.35 \pm 0.031$ & $5.09 \pm 0.036$ \\
SHOC588 & $5.73 \pm 0.032$ & $6.35 \pm 0.029$ & $1.22 \pm 0.041$ & $5.80 \pm 0.031$ & $5.09 \pm 0.089$ \\
SHOC036 & $5.22 \pm 0.033$ & $5.91 \pm 0.031$ & $1.77 \pm 0.16$ & $5.25 \pm 0.049$ & $5.16 \pm 0.099$ \\
SHOC575 & $5.74 \pm 0.037$ & $6.48 \pm 0.031$ & - & $5.95 \pm 0.036$ & - \\
SHOC579 & $5.54 \pm 0.014$ & $6.36 \pm 0.015$ & $1.31 \pm 0.016$ & $5.76 \pm 0.013$ & $5.19 \pm 0.020$ \\
FTDTR-8 & $5.14 \pm 0.040$ & $5.89 \pm 0.032$ & $1.86 \pm 0.082$ & $5.42 \pm 0.042$ & $5.37 \pm 0.059$ \\
SHOC263 & $6.00 \pm 0.046$ & $6.60 \pm 0.049$ & - & $5.87 \pm 0.044$ & - \\
FTDTR-9 & $5.74 \pm 0.083$ & $6.52 \pm 0.069$ & - & $5.73 \pm 0.081$ & - \\
FTDTR-10 & $5.52 \pm 0.057$ & $6.18 \pm 0.048$ & - & $5.46 \pm 0.056$ & - \\
\hline & & & & &
\end{tabular}


Table 7. Element abundances and helium mass fractions using either oxygen $\left(Y_{O / H}\right)$ or sulphur $\left(Y_{S / H}\right)$ in eq. 30.

\begin{tabular}{|c|c|c|c|c|c|c|}
\hline HII Galaxy & $\mathrm{He} / \mathrm{H}$ & $Y_{(O / H)}$ & $Y_{(S / H)}$ & $12+\log (\mathrm{O} / \mathrm{H})$ & $12+\log (N / H)$ & $12+\log (\mathrm{S} / \mathrm{H})$ \\
\hline FTDTR-1 $^{\mathrm{O}, \mathrm{N}, \mathrm{S}}$ & $0.0977 \pm 0.0011$ & $0.283 \pm 0.0023$ & $0.283 \pm 0.0024$ & $7.88 \pm 0.064$ & $5.97 \pm 0.039$ & $6.20 \pm 0.047$ \\
\hline $\mathrm{IZw} 18^{\mathrm{O}}, \mathrm{N}, \mathrm{S}$ & $0.0768 \pm 0.0028$ & $0.236 \pm 0.0065$ & $0.236 \pm 0.0064$ & $7.08 \pm 0.082$ & $5.34 \pm 0.038$ & $5.34 \pm 0.055$ \\
\hline MRK36-A1 $\mathrm{O}, \mathrm{N}, \mathrm{S}$ & $0.0795 \pm 0.0082$ & $0.245 \pm 0.019$ & $0.244 \pm 0.019$ & $7.87 \pm 0.059$ & $6.11 \pm 0.21$ & $6.15 \pm 0.034$ \\
\hline MRK36-A2 $\mathrm{O}, \mathrm{N}, \mathrm{S}$ & $0.0801 \pm 0.0097$ & $0.244 \pm 0.022$ & $0.246 \pm 0.023$ & $7.98 \pm 0.033$ & $5.97 \pm 0.33$ & $6.32 \pm 0.020$ \\
\hline $\mathrm{MRK} 475^{\mathrm{O}}, \mathrm{N}, \mathrm{S}$ & $0.0883 \pm 0.0045$ & $0.264 \pm 0.0096$ & $0.265 \pm 0.0098$ & $8.00 \pm 0.037$ & $6.25 \pm 0.25$ & $6.37 \pm 0.023$ \\
\hline FTDTR-2 $2^{\mathrm{S}}$ & $0.0756 \pm 0.0033$ & - & $0.235 \pm 0.0080$ & - & - & $6.24 \pm 0.057$ \\
\hline $\mathrm{IZw} 70^{\mathrm{O}}, \mathrm{N}, \mathrm{S}$ & $0.0943 \pm 0.0032$ & $0.274 \pm 0.0067$ & $0.275 \pm 0.0068$ & $8.38 \pm 0.086$ & $6.64 \pm 0.028$ & $6.52 \pm 0.038$ \\
\hline MRK689 & $0.0775 \pm 0.0068$ & $0.235 \pm 0.016$ & $0.236 \pm 0.016$ & $8.57 \pm 0.076$ & $6.54 \pm 0.025$ & $6.66 \pm 0.034$ \\
\hline $\mathrm{MRK} 67^{\mathrm{O}}, \mathrm{N}, \mathrm{S}$ & $0.0910 \pm 0.012$ & $0.267 \pm 0.026$ & $0.269 \pm 0.025$ & $8.21 \pm 0.044$ & $6.31 \pm 0.19$ & $6.42 \pm 0.030$ \\
\hline FTDTR-3 $3^{\mathrm{O}}, \mathrm{N}, \mathrm{S}$ & $0.0847 \pm 0.0030$ & $0.252 \pm 0.0067$ & $0.253 \pm 0.0069$ & $8.20 \pm 0.038$ & $6.35 \pm 0.24$ & $6.48 \pm 0.025$ \\
\hline $\mathrm{SHOC} 022^{\mathrm{O}}, \mathrm{N}, \mathrm{S}$ & $0.0838 \pm 0.0018$ & $0.253 \pm 0.0041$ & $0.254 \pm 0.0040$ & $8.27 \pm 0.073$ & $6.55 \pm 0.040$ & $6.61 \pm 0.040$ \\
\hline FTDTR- $4^{\mathrm{O}, \mathrm{N}, \mathrm{S}}$ & $0.0878 \pm 0.0015$ & $0.261 \pm 0.0033$ & $0.262 \pm 0.0032$ & $8.16 \pm 0.059$ & $6.34 \pm 0.021$ & $6.42 \pm 0.036$ \\
\hline SHOC220 & $0.0989 \pm 0.0023$ & $0.287 \pm 0.0046$ & $0.287 \pm 0.0047$ & $7.60 \pm 0.022$ & $6.36 \pm 0.079$ & $5.89 \pm 0.019$ \\
\hline FTDTR-5 ${ }^{\mathrm{S}}$ & $0.0779 \pm 0.0042$ & - & $0.238 \pm 0.010$ & - & - & $6.37 \pm 0.073$ \\
\hline FTDTR-6 ${ }^{\mathrm{O}}, \mathrm{N}, \mathrm{S}$ & $0.0869 \pm 0.0019$ & $0.259 \pm 0.0043$ & $0.260 \pm 0.0042$ & $8.22 \pm 0.045$ & $6.26 \pm 0.16$ & $6.51 \pm 0.029$ \\
\hline FTDTR- $7^{\mathrm{O}}, \mathrm{N}, \mathrm{S}$ & $0.0864 \pm 0.0013$ & $0.257 \pm 0.0028$ & $0.258 \pm 0.0028$ & $8.16 \pm 0.019$ & $6.39 \pm 0.027$ & $6.38 \pm 0.014$ \\
\hline $\mathrm{MRK} 627^{\mathrm{O}}, \mathrm{N}, \mathrm{S}$ & $0.0845 \pm 0.0040$ & $0.251 \pm 0.0089$ & $0.252 \pm 0.0085$ & $8.52 \pm 0.096$ & $6.77 \pm 0.032$ & $6.66 \pm 0.042$ \\
\hline SHOC592 & $0.0957 \pm 0.0079$ & $0.275 \pm 0.016$ & $0.276 \pm 0.017$ & $8.32 \pm 0.048$ & $7.13 \pm 0.025$ & $6.86 \pm 0.044$ \\
\hline PHL293B ${ }^{\mathrm{O}, \mathrm{S}}$ & $0.0766 \pm 0.0024$ & $0.238 \pm 0.0056$ & $0.238 \pm 0.0057$ & $7.74 \pm 0.020$ & - & $6.21 \pm 0.025$ \\
\hline SHOC588 & $0.0940 \pm 0.0084$ & $0.273 \pm 0.018$ & $0.273 \pm 0.018$ & $8.25 \pm 0.025$ & $7.12 \pm 0.029$ & $6.53 \pm 0.028$ \\
\hline SHOC036 & $0.102 \pm 0.010$ & $0.293 \pm 0.020$ & $0.292 \pm 0.020$ & $7.74 \pm 0.038$ & $6.55 \pm 0.049$ & $6.24 \pm 0.051$ \\
\hline SHOC575 & $0.0977 \pm 0.0018$ & $0.280 \pm 0.0038$ & $0.281 \pm 0.0038$ & $8.28 \pm 0.049$ & $7.37 \pm 0.026$ & $6.55 \pm 0.032$ \\
\hline SHOC579 & $0.0991 \pm 0.0049$ & $0.284 \pm 0.0099$ & $0.284 \pm 0.0098$ & $8.11 \pm 0.0057$ & $7.28 \pm 0.0082$ & $6.54 \pm 0.013$ \\
\hline FTDTR- $8^{\mathrm{O}}, \mathrm{N}, \mathrm{S}$ & $0.0750 \pm 0.0018$ & $0.230 \pm 0.0041$ & $0.231 \pm 0.0042$ & $7.93 \pm 0.050$ & $6.09 \pm 0.11$ & $6.23 \pm 0.041$ \\
\hline $\mathrm{SHOC} 263^{\mathrm{O}}, \mathrm{N}, \mathrm{S}$ & $0.0866 \pm 0.0056$ & $0.256 \pm 0.012$ & $0.257 \pm 0.013$ & $8.42 \pm 0.085$ & $6.87 \pm 0.041$ & $6.69 \pm 0.046$ \\
\hline FTDTR-9O, N, S & $0.0890 \pm 0.0032$ & $0.265 \pm 0.0068$ & $0.266 \pm 0.0064$ & $8.33 \pm 0.12$ & $6.62 \pm 0.069$ & $6.58 \pm 0.071$ \\
\hline FTDTR- $10^{\mathrm{O}}, \mathrm{N}, \mathrm{S}$ & $0.0783 \pm 0.0026$ & $0.240 \pm 0.0060$ & $0.240 \pm 0.0058$ & $7.89 \pm 0.080$ & $6.48 \pm 0.21$ & $6.27 \pm 0.048$ \\
\hline
\end{tabular}

The superscript on the names indicates the $\mathrm{Y}, \mathrm{Z}$ regressions, where the object is included.

relation was also found by Pagel et al. (1992) and confirmed by Rosales-Ortega (2006). This was an unexpected result given the primary/secondary nature of $\mathrm{N}$ : the $\mathrm{C}-\mathrm{N}-\mathrm{O}$ cycle from low mass stars and the $\alpha$ process in massive stars. In contrast, the oxygen and sulphur nucleosynthesis can only be originated by the second mechanism. It has been claimed that the agreement between the $\mathrm{Y}-\mathrm{O}$ and $\mathrm{Y}-\mathrm{N}$ regressions is expected under the premises: no serious pollution from Wolf-Rayet stars and low metallicities $\left(N / H<6.6 \cdot 10^{-6}\right)$. The He-S regression proposed in this paper is also fairly linear for the metallicity range of our sample. Despite the predicted intrinsic linearity in the chemical evolution between helium and these heavy elements, some objects seem to be clear outliers in the relation. In what follows we propose reasons that could explain these outliers.

The top panel of Fig. 9 shows the regression of helium vs oxygen abundances. Indicated are the objects that were excluded from the fit. The galaxies with the higher $\mathrm{O} / \mathrm{H}$ abundance appear to display a lower than expected Y abundance. All these objects were observed with Configuration I, which does not reach the [OII] $\lambda \lambda 3726 \AA, 3729 \AA$ doublet. Consequently, in these low ionization objects, where $\mathrm{O}^{+} / \mathrm{H}^{+}>\mathrm{O}^{++} / \mathrm{H}^{+}$there is a greater uncertainty in the $O^{+}$as its value depends on weak lines. The temperatures deduced for them are also the lowest of the sample. Among them is MRK689, having the largest error bars both in $O$ and in $Y$ and for which we derived the highest $O / H$ value, so this object weights heavily in the linear fit for the regression. For all these reasons, we did not include it in the fit. Below we discuss other objects that were excluded from the final regression.

The middle panel of Fig. 9 shows the helium vs. nitrogen regression. Two objects, SHOC220 and PHL293, show indications of complex kinematics. PHL293 has been extensively analyzed by Terlevich et al. (2014). Its spectrum shows two very broad $(\sim 1000 \mathrm{~km} / \mathrm{s}$ and $\sim 4000 \mathrm{~km} / \mathrm{s}) \mathrm{H \alpha}$ components which the authors argue are generated by a young and dense expanding supershell or a stationary cooling wind, both driven by the young cluster wind. The galaxy SHOC220 displays similarly intense wide $H \alpha$ components. Since these broad components, overlap the [NII] $\lambda \lambda 6548 \AA, 6583 \AA$ lines, these galaxies were excluded from the $Y-N$ regression.

Finally, the bottom panel shows the $\mathrm{Y}$ vs. S regression where all objects are included even when for several objects the telluric correction for the $[S I I I] \lambda \lambda 9069,9532 \AA$ lines could not be performed. This can be appreciated on the observed ratios between these lines in Table 4. For these objects, the theoretical relation between both lines was used based on the line of the doublet that falls on the least perturbed sky region. SHOC036 and SHOC263 were observed during adverse conditions and none of the [SIII] lines were detected.

In all three panels in Fig. 9 a few objects appear to show a helium abundance distinctively above the linear fit. These Yenhanced objects occupy a different position for each regression:. They fall in the low metallicity edge of the $\mathrm{He}-\mathrm{O}$ plot bue on the high metallicity one for the $\mathrm{He}-\mathrm{N}$ plot. Fig. 10 shows the $N / O$ ratio versus $y$. The majority of the objects occupy a narrow band, $\Delta N / O \approx 0.025$ of about but for those objects with $y>0.09$, the $N / O$ ratio has a larger dispersion. This phenomenon is observed in both the low and high metallicity galaxies. Three of these objects: SHOC575, SHOC579 and SHOC022, are identified as Wolf-Rayet galaxies (WR038, WR039 and WR057) in Brinchmann et al. (2008). Detecting WR features using single slit observations is a challenging task, since the slit must be precisely located in order to find the stars. Even the well observed galaxy $I Z w 18$ defied WR detection for a long time, until the work by Izotov et al. (1997) and Legrand et al. (1997). These three WR galaxies are included in Pérez-Montero et al. (2013) in their study of the pollution of the interstellar medium by WR stars. Our derived values $\log (N / O)=-0.91 \pm 0.05,-0.82 \pm 0.1,-1.71 \pm 0.08$ 

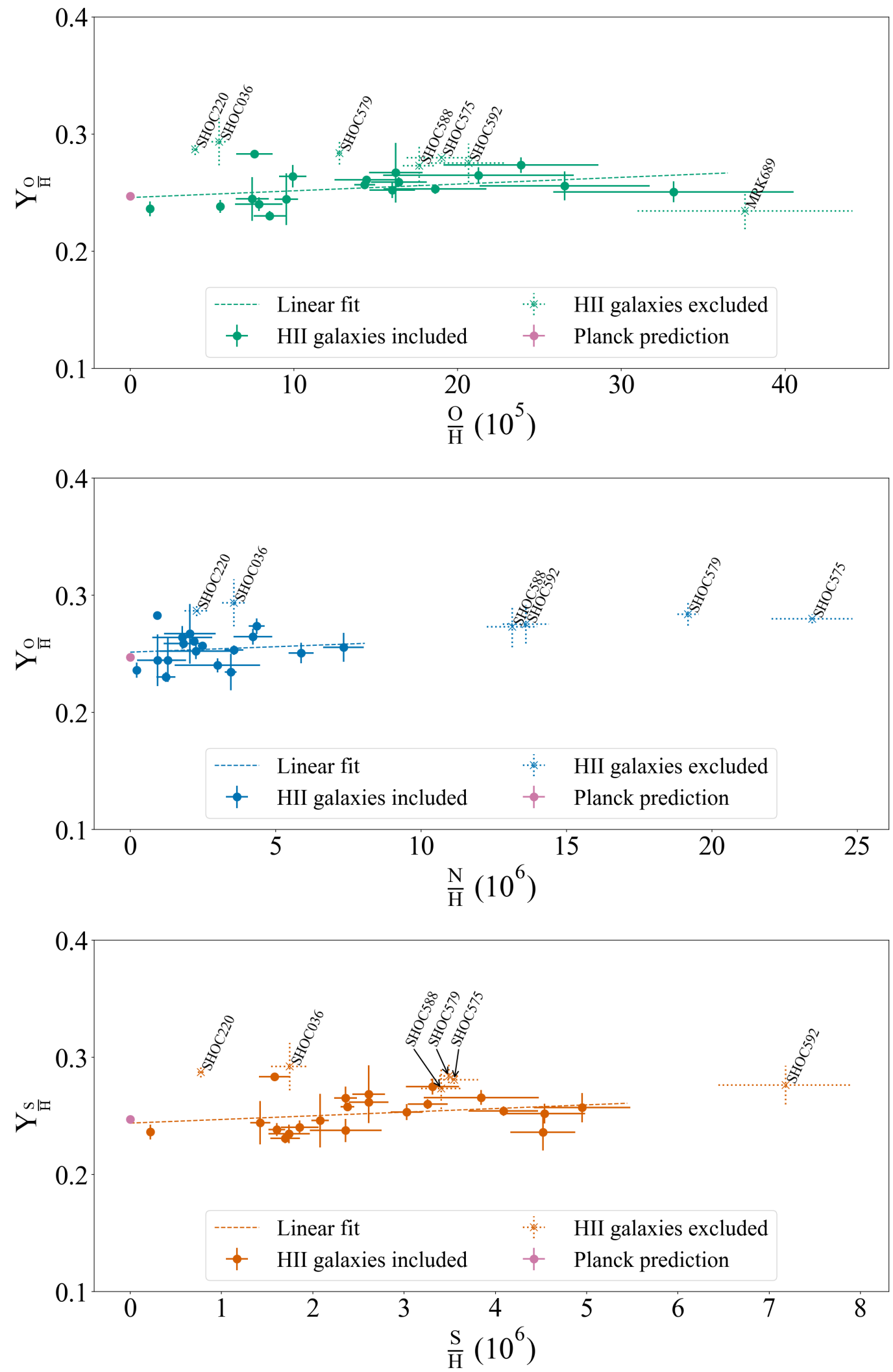

Figure 9. Primordial helium linear regressions using oxygen, nitrogen and sulphur as metallicity tracer. 


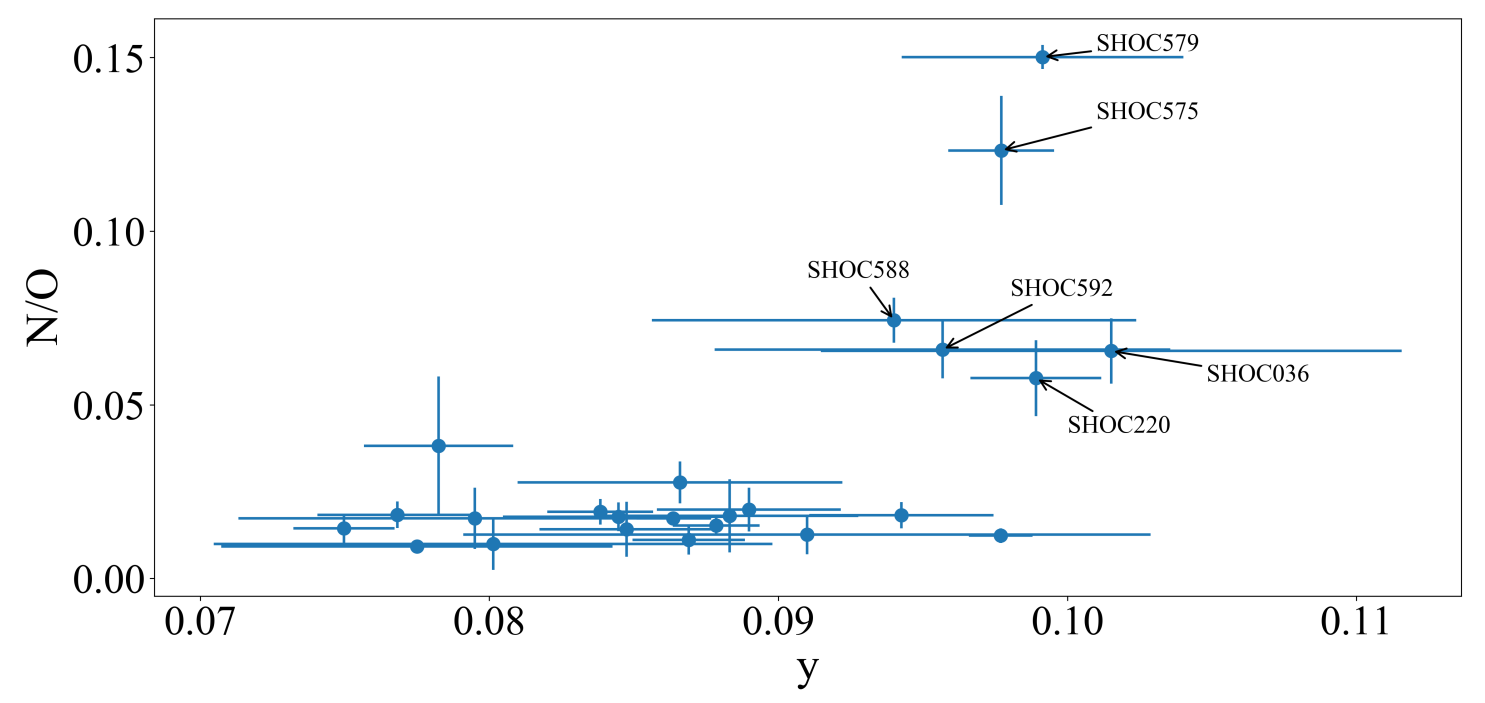

Figure 10. Nitrogen to oxygen ratio versus helium abundance for our sample. The labeled objects have been excluded from the linear regressions as outliers (see text).

agree within uncertainties with theirs $\log (N / O)=0.87 \pm 0.27$, $-0.80 \pm 0.20,-1.48 \pm 0.26$ obtained on the basis of integral field spectroscopy. Pérez-Montero et al. (2013) concluded that while the oxygen abundance in these galaxies is uniform in scales of the order of several kpc, the $N / O$ ratio is not homogeneous. Higher nitrogen excess was found at the spaxels close to the WR. Further study, beyond the scope of this work, will probably determine how these objects attained their helium enrichment, but it is clear that these objects have an impact on the regressions. On the $Y-O$ fit these galaxies tend to increase the $Y_{P}$ extrapolation while on the $Y-N$ relation they decrease it. Consequently, we have excluded the six galaxies with $\mathrm{N} / \mathrm{O}$ excess (see Fig. 10) from all the $Y_{\boldsymbol{P}}$ linear regressions. These are: SHOC579, SHOC575, SHOC588, SHOC592, SHOC036 and SHOC220. SHOC022 and IZwicky18 are still included even when they show the presence of WR stars. As they don't present any anomaly in their $N / O$ values, we assumed that our observations do not include contaminated regions. In the $Y-S$ regression these objects seem to have a helium enrichment. In contrast, their sulfur abundances do not seem to diverge from the rest of the sample, unlike in the oxygen and nitrogen cases. For consistency, however, these objects were excluded as outliers from all the linear regressions. Still, they have been included in Fig. 9 with dashed uncertainty bars.

\subsection{Linear regression}

The helium abundance, helium mass fraction and the abundances of $\mathrm{O}, \mathrm{N}$ and $\mathrm{S}$ are given in Table 7. Two helium mass fractions were calculated for each object. From the mass fraction formula $(X+Y+Z=1)$ it can be derived that:

$Y=\frac{4 \frac{H e I}{H I}(1-Z)}{1+4 \frac{H e I}{H I}}$

where it is common to express $\mathrm{Z}$ as a function of the oxygen abundance: $Z \sim 20 O / H$ or a similar expression using $N$ (see eq. 28 ). Izotov et al. (2013) suggest a modification of the classical expression using an empirical oxygen-metallicity relation, $Z=f(O / Z)$. In order to use sulphur as an alternative metallicity tracer we define:

$Z \sim 20 \frac{O}{S} \cdot \frac{S}{H}$

The oxygen to sulphur ratio was studied by Díaz et al. (1991) for the high metallicity star forming regions in $M 51$. Their result was confirmed recently by Dors et al. (2016) for a large sample of Blue Compact Dwarfs. They obtain $\langle\log (S / O)\rangle=-1.53 \pm 0.03$ and $\langle\log (S / O)\rangle=-1.78 \pm 0.02$ for high and low metallicity HII regions respectively. The low metallicity value is used in eq. 31 for our objects. The Y values calculated using $\mathrm{O}$ or $\mathrm{S}$ (see Table 8) differ on a slightly larger dispersion when using $S$, where the error on the $S / O$ gradient is taken into account. To compute the linear regression we used the curvefit routine in the scipy package (Jones et al. 2001-) within a bootstrap algorithm: in a ten thousand iterations loop, a linear fit was performed on the helium mass fractions and heavy element abundances, generated from a normal distribution for each galaxy. This process was repeated for each $Y-Z$ combination. From the output distributions, we determined the mean $Y_{P, Z}$ and the $1 \sigma$ uncertainty for each element regression.

The first three rows in Table 8 show the primordial helium abundance determinations using one of the three elements as metallicity proxy. Column (3) in this table displays the number of objects included in that regression. A good agreement is obtained from the three regressions: $Y_{P}, O=0.246 \pm 0.005$, $Y_{P, N}=0.251 \pm 0.005, Y_{P}, S=0.244 \pm 0.006$ within their uncertainty. By using $\mathrm{O}$ and $\mathrm{S}$ as metallicity proxy to determine $Y_{P}$, we obtain similar values, while using $\mathrm{N}$ results in a slightly larger $Y_{P}$. Limiting the regressions to the 17 objects in our sample having oxygen, nitrogen and sulfur abundance determinations we get slightly larger mean values: $Y_{P, O}=0.248 \pm 0.006, Y_{P, N}=0.252 \pm 0.005$ and $Y_{P, S}=0.246 \pm 0.006$. As already discussed, most of the sample for this work has been obtained with observations designed ad-hoc to measure the [SII] and [SIII] lines, and as a consequence our preferred regression is the one obtained using $\mathrm{S}$ :

$Y_{P, S}=0.244 \pm 0.006$

We obtain good agreement within uncertainties with the most recent work on the topic using a $Y-O$ regression. The latest result from Peimbert et al. (2016) is $Y_{P, O}=0.2446 \pm 0.0029$ including the effect of temperature fluctuations (that would decrease $Y_{P}$ by a value between 0.003 and 0.006 ). The MCMC helium abundance determination from Aver et al. (2015) gives $Y_{P, O}=0.2449 \pm 0.004$ using observations and the oxygen abundance calculation from Izotov et al. (2014). Izotov and colleagues obtain $Y_{P, O}=0.2551 \pm 0.0022$, a higher value which they claim it might require non-standard physics at the time of Big Bang nucle- 
Table 8. Primordial helium abundance determinations from linear regression combinations and comparison with the literature.

\begin{tabular}{lcc}
\hline Elemental regression & Magnitude & Number of objects \\
\hline$Y_{P, O}$ & $0.246 \pm 0.005$ & 18 \\
\hline$Y_{P, N}$ & $0.251 \pm 0.005$ & 18 \\
\hline$Y_{P, S}$ & $0.244 \pm 0.006$ & 21 \\
\hline$Y_{P, O-N}$ & $0.247 \pm 0.006$ & 17 \\
\hline$Y_{P, O-S}$ & $0.244 \pm 0.006$ & 18 \\
\hline$Y_{P, N-S}$ & $0.250 \pm 0.007$ & 18 \\
\hline$Y_{P, O-N-S}$ & $0.245 \pm 0.007$ & 17 \\
\hline \hline$Y_{P, O}^{1}, 0.2446 \pm 0.0029$ & 5 \\
\hline$Y_{P, O}^{2}$ & $0.2449 \pm 0.004$ & 15 \\
\hline$Y_{P, O}^{3}$ & $0.2551 \pm 0.0022$ & 28 \\
\hline$Y_{P, P \text { lanckBBN }}^{4}$ & $0.24467 \pm 0.0002$ & - \\
\hline
\end{tabular}

[1] Peimbert et al. (2016) [2] Aver et al. (2015) [3] Izotov et al. (2014) [4] Planck Collaboration et al. (2016) (This value represents an upper limit from the four $\Lambda C D M$ parameter configurations presented by the authors)

osynthesis. Their regression includes the largest number of objects and their observations reached the near-IR HeI $10830 \AA$ line. Assuming a standard cold dark matter paradigm the Planck collaboration provides a (model dependent) value of $Y_{P, B B N \text { Planck }}=$ $0.24467 \pm 0.0002$ (see Planck Collaboration et al. 2016). All three regressions agree with this result, within their errors. The mean values obtained both using $S$ and $O$ are closer to the Planck value, while we obtain a larger $Y_{P}$ value using $N$. This could be due to a selective $\mathrm{N}$ enrichment in these objects, but chemical evolution corrections fall beyond our analysis, on account of the uncertainties in all $Y_{P, Z}$ determinations.

It may be appreciated from this analysis, that deviant objects both in $\mathrm{Y}$ and $\mathrm{Z}$ have a large impact in the regression. This is partly due to the restricted range in metallicity for our sample objects. It would be interesting to include in the future higher metallicity objects (see Carigi \& Peimbert 2010) even when for such cases deriving the $\mathrm{O}$ abundance will become more difficult (see, e.g. Hägele et al. 2008). One of the advantages of including sulphur is that its abundance can be measured with reasonable accuracy in metal rich, lower ionization HII regions, increasing the baseline for the linear fit.

As discussed by Peimbert et al. (2007b) the third most important source of error in determining $Y_{P}$ lies in the extrapolation of the $\mathrm{Y}$ values to zero metallicity. To add further insight into this uncertainty source, we propose to apply a multiple/multivariable linear regression model. In this framework, a linear relation is established between multiple independent variables and a dependent variable expressed as a scalar. The approach is valid for this analysis as the abundances involved are independent. In these fittings, we employ the helium fraction by mass calculated using sulfur $\left(Y=Y_{S}\right)$ and we apply a similar boot-strap algorithm to estimate $Y_{\boldsymbol{P}}$. The possible combinations are displayed in Table 8. Even though including additional elements does not seem to reduce the uncertainty, the predicted $Y_{P}$ agrees better with the results by Aver et al. (2015) and Peimbert et al. (2016). This is particularly true for the $Y_{P, O-N-S}=0.245 \pm 0.007$ regression. This result vindicates the addition of more elements as metallicity tracers, in particular sulphur. In a forthcoming paper we plan to apply MCMC sampling to the $Y-Z$ regressions in order to explore how the addition of more elements affects the $Y_{P}$ calculation.

\section{CONCLUSIONS}

We observed with ISIS in the WHT in two different configurations, 27 low metallicity young HII galaxies selected from SDSS - DR12. The objective was to reach the near-IR [SIII] $\lambda \lambda 9069,9532 \AA$ lines to estimate $T[S I I I]$, the abundances of $\mathrm{S}$ and He and to derive $Y_{\boldsymbol{P}}$, the primordial abundance of helium from $d Y / d Z$ using for the first time $S$ as a tracer of metal abundance.

The selected sample with $(E W(H \alpha)>200 \AA)$ represents very young bursts. In these objects, the nebular continuum contributes as much as the youngest stellar population to the continuum and needs to be taken into account.

The nebular continuum was calculated from first principles and removed from the observed spectra. The helium abundance was derived using the direct method. The underlying absorption on the recombination lines was subtracted using a SSP fit which provided a complete characterization of the stellar population for the spectra obtained with the instrumental setup that covered a wide spectral base. For the configuration that covered a smaller wavelength range, the older stellar population was more difficult to characterize and subtract. We hope this will improve in the near future with stellar bases which go beyond the $7000 \AA$ boundary.

We could determine from our data two temperature diagnostics: $T[O I I I]$ and $T[S I I I]$. A model with two ionization zones was considered. When the errors for both temperatures were significantly different, an empirical relation was used to derive the temperature from the more precise determination and the error was propagated.

We determined the ionic and total abundances for oxygen and nitrogen. For sulphur, in high ionization zones the $S^{3+}$ component cannot be neglected. We proposed an ICF $\left(S^{3+}\right)$ based on the $A r^{3+} / A r^{2+}$ ratio, appropriate for our wavelength range. This was derived using Cloudy photo-ionization models (Ferland et al. 2013) with PopStar evolutionary models Martín-Manjón et al. (2010).

Linear regressions were found between $\mathrm{Y}$ and $\mathrm{O}, \mathrm{Y}$ and $\mathrm{N}$ and Y and S. Six objects: SHOC036, SHOC220, SHOC592, SHOC588, SHOC575 and SHOC579 show helium excess. Three of them are known to have WR stars probably contaminating the interstellar medium. These objects also show a nitrogen to oxygen excess $(N / O>0.04)$, and $(H e / H>0.09)$. They were excluded from the seven linear regressions.

The results for primordial helium from the three regressions are $Y_{P, O}=0.246 \pm 0.005, Y_{P, N}=0.252 \pm 0.005, Y_{P, S}=$ $0.244 \pm 0.006$; they agree with each other within errors and also with the derived value from Planck within standard Big Bang Nucleosynthesis prescriptions. A multivariable regression using all three elements results in $Y_{O-N-S}=0.245 \pm 0.007$. The novel use of $\mathrm{S}$ as proxy for the metal abundance and to derive $Y_{P}$ has proved successful.

In a forthcoming paper (Fernández et al. in preparation) we will include true error propagation between all the steps involved to correct better the telluric features and to improve the sulphur abundance determination. We are also improving the fit for the underlying stellar population and follow Izotov et al. (2014) and Aver et al. (2015) multidimensional schemes to compute the helium abundance. This procedure can simultaneously quantify the different processes contributing to the emission in the helium lines.

As future improvements to the programe, we plan to include in forthcoming observation campaigns, the relatively strong HeI $110830 \AA$ line with high sensitivity to electron density and obvious advantage to derive helium abundance (see Izotov et al. 2014; Aver et al. 2015). We will also use new stellar libraries that extend up to near-infrared wavelengths to subtract better the stellar population contamination to the emission line spectrum. We 
are aware of tools such as skycorr developed by Noll et al. (2014), which can model and remove airglow contamination. This contribution is instrument-independent and it will allow in the future to propagate the error in the sky subtraction procedure.

\section{ACKNOWLEDGMENTS}

We are thankful to Enrique Pérez-Montero and Daniel Miralles for their generous discussions on the nebular and stellar continua determination and to Manuel Emilio Moreno Raya for his company during the first observation on the William Herschel telescope.

The authors also wish to thank an anonymous referee whose comments helped to improve the clarity of the paper.

We thank the Spanish allocation committee (CAT) for awarding observing time and the cheerful technical support from the observatory personnel. Vital Fernández is grateful to the Mexican research Council (CONACYT) for suporting this research through studenship 554031/300844. Elena Terlevich and Roberto Terlevich acknowledge CONACYT for supportig this research under grants: CB-2008-103365. This work has been supported by DGICYT grants AYA2013-47742-C4-3-P and AYA2016-79724C4-1-P. Partial financial support came also from proyect SELGIFS: PIRSES-GA-2013-612701-SELGIFS. Vital Fernández is grateful to the hospitality of the Departamento de Física Teórica at the Universidad Autónoma de Madrid, Spain.

The WHT is operated in the island of La Palma by the Isaac Newton Group in the Spanish Observatorio del Roque de los Muchachos of the Instituto de Astrofísica de Canarias.

Funding for the creation and distribution of the SDSS Archive has been provided by the Alfred P. Sloan Foundation, the Participating Institutions, the National Aeronautics and Space Administration, the National Science Foundation, the US Department of Energy, the Japanese Monbukagakusho, and the Max Planck Society. The SDSS Web site is http://www.sdss.org.

This research has made use of the NASA/IPAC Extragalactic Database (NED) which is operated by the Jet Propulsion Laboratory California Institute of Technology, under contract with the National Aeronautics and Space Administration.

\section{References}

Aggarwal K. M., Keenan F. P., 2000, VizieR Online Data Catalog, 212

Ahn C. P. et al., 2014, The Astrophysical Journal Supplement Series, 211, 17

Aver E., Olive K. A., Skillman E. D., 2010, Journal of Cosmology and Astroparticle Physics, 2010, 003

Aver E., Olive K. A., Skillman E. D., 2015, Journal of Cosmology and Astroparticle Physics, 7, 011

Baldwin J. A., Phillips M. M., Terlevich R., 1981, Publications of the Astronomical Society of the Pacific, 93, 5

Berg D. A., Skillman E. D., Croxall K. V., Pogge R. W., Moustakas J., Johnson-Groh M., 2015, The Astrophysical Journal, 806,16

Brinchmann J., Kunth D., Durret F., 2008, Astronomy \& Astrophysics, 485, 657, arXiv: 0805.1073

Brown R. L., Mathews W. G., 1970, Astrophysical Journal, 160, 939

Bruzual G., Charlot S., 2003, Monthly Notices of the Royal Astronomical Society, 344, 1000

Burles S., Tytler D., 1998, The Astrophysical Journal, 499, 699

Carigi L., Peimbert M., 2010, arXiv preprint arXiv:1004.0756

Carswell R. F., Rauch M., Weymann R. J., Cooke A. J., Webb J. K., 1994, Monthly Notices of the Royal Astronomical Society, 268, L1
Cid Fernandes R., Mateus A., Sodré L., Stasińska G., Gomes J. M., 2005, 363-378

Cid Fernandes R. et al., 2013, Astronomy and Astrophysics, 557, 86

Croxall K. V., Pogge R. W., Berg D., Skillman E. D., Moustakas J., 2015, arXiv:1501.02272 [astro-ph]

Davidson K., Kinman T. D., 1985, The Astrophysical Journal Supplement Series, 58, 321

Díaz A. I., Pagel B. E. J., Wilson I. R. G., 1985, Monthly Notices of the Royal Astronomical Society, 212, 737

Díaz A. I., Terlevich E., Vílchez J. M., Pagel B. E. J., Edmunds M. G., 1991, Monthly Notices of the Royal Astronomical Society, 253, 245

Dors O., Pérez-Montero E., Hägele G., Cardaci M., Krabbe A., 2016, Monthly Notices of the Royal Astronomical Society, 456, 4407

Dottori H. A., Bica E. L. D., 1981, Astronomy and Astrophysics, 102,245

Dufton P. L., Hibbert A., Kingston A. E., Doschek G. A., 1982, The Astrophysical Journal, 257, 338

Ercolano B., Storey P. J., 2006, Monthly Notices of the Royal Astronomical Society, 372, 1875-1878

Ferland G. J. et al., 2017, Revista Mexicana de Astronomía y Astrofísica, 53, 385

Ferland G. J. et al., 2013, 137

Fukugita M., Kawasaki M., 2006, The Astrophysical Journal, 646, 691

Galavís M. E., Mendoza C., Zeippen C. J., 1995, Astronomy and Astrophysics Supplement Series, 111, 347

Galavís M. E., Mendoza C., Zeippen C. J., 1997, Astronomy and Astrophysics Supplement Series, 123, 13

García-Rojas J., Peña M., Morisset C., Mesa-Delgado A., Ruiz M. T., 2012, Astronomy and Astrophysics, 538, A54

Garnett D. R., 1992, The Astronomical Journal, 103, 1330

Gomes J. M., Papaderos P., 2017, Astronomy and Astrophysics, 603, A63

González-Delgado R. M., Cervino M., Martins L. P., Leitherer C., Hauschildt P. H., 2005, Monthly Notices of the Royal Astronomical Society, 357, 945

Gordon K. D., Clayton G. C., Misselt K. A., Landolt A. U., Wolff M. J., 2003, The Astrophysical Journal, 594, 279

Hägele G. F., Díaz A. I., Terlevich E., Terlevich R., Pérez-Montero E., Cardaci M. V., 2008, Monthly Notices of the Royal Astronomical Society, 383, 209

Hägele G. F., Pérez-Montero E., Diaz A. I., Terlevich E., Terlevich R., 2006, Monthly Notices of the Royal Astronomical Society, 372, 293, arXiv: astro-ph/0608244

Hudson C. E., Ramsbottom C. A., Scott M. P., 2012, The Astrophysical Journal, 750, 65

Izotov Y., Schaerer D., Blecha A., Royer F., Guseva N., North P., 2006, Astronomy \& Astrophysics, 459, 71, arXiv: astro$\mathrm{ph} / 0608203$

Izotov Y. I., Foltz C. B., Green R. F., Guseva N. G., Thuan T. X., 1997, The Astrophysical Journal Letters, 487, L37

Izotov Y. I., Stasińska G., Guseva N. G., 2013, Astronomy and Astrophysics, 558, A57

Izotov Y. I., Thuan T. X., Guseva N. G., 2014, Monthly Notices of the Royal Astronomical Society, 445, 778

Johnson C. T., Kingston A. E., Dufton P. L., 1986, Monthly Notices of the Royal Astronomical Society, 220, 155

Jones E., Oliphant T., Peterson P., et al., 2001-, SciPy: Open source scientific tools for Python

Kaufman V., Sugar J., 1986, Journal of Physical and Chemical Reference Data, 15, 321

Kehrig C. et al., 2016, Monthly Notices of the Royal Astronomical Society, 459, 2992

Kennicutt R. C., Bresolin F., Garnett D. R., 2003, The Astrophysical Journal, 591, 801 
Kniazev A. Y., Grebel E. K., Hao L., Brinkmann J., Fukugita M., 2003, The Astrophysical Journal Letters, 593, L73

Legrand F., Kunth D., Roy J., Mas-Hesse J. M., Walsh J. R., 1997, Astronomy and Astrophysics, 326, L17

Leitherer C. et al., 1999, The Astrophysical Journal Supplement Series, 123, 3

Lequeux J., Peimbert M., Rayo J. F., Serrano A., Torres-Peimbert S., 1979, Astronomy and Astrophysics, 80, 155

Liu X., Luo S., Barlow M. J., Danziger I. J., Storey P. J., 2001, Monthly Notices of the Royal Astronomical Society, 327, 141

Liu X., Storey P. J., Barlow M. J., Danziger I. J., Cohen M., Bryce M., 2000, Monthly Notices of the Royal Astronomical Society, 312, 585

Luridiana V., Morisset C., Shaw R. A., 2015, Astronomy and Astrophysics, 573, A42

Luridiana V., Peimbert A., Peimbert M., Cerviño M., 2003, The Astrophysical Journal, 592, 846

Martín-Manjón M. L., García-Vargas M. L., Mollá M., Díaz A. I., 2010, Monthly Notices of the Royal Astronomical Society, 403, 2012

Mendoza C., Zeippen C. J., 1982, Monthly Notices of the Royal Astronomical Society, 198, 127

Mollá M., García-Vargas M. L., Bressan A., 2009, Monthly Notices of the Royal Astronomical Society, 398, 451

Noll S., Kausch W., Kimeswenger S., Barden M., Jones A. M., Modigliani A., Szyszka C., Taylor J., 2014, Astronomy \& Astrophysics, 567, A25

Nussbaumer H., Schmutz W., 1984, Astronomy and Astrophysics, 138,495

Olive K. A., Skillman E. D., 2004, The Astrophysical Journal, 617, 29

Osterbrock D. E., 1974, Astrophysics of gaseous nebulae

Pagel B. E. J., Simonson E. A., Terlevich R. J., Edmunds M. G., 1992, Monthly Notices of the Royal Astronomical Society, 255, 325

Peimbert A., Peimbert M., Luridiana V., 2002, 668

Peimbert A., Peimbert M., Luridiana V., 2016, Revista Mexicana de Astronomía y Astrofísica, 52, 419

Peimbert M., Costero R., 1969, Boletín de los Observatorios Tonantzintla y Tacubaya, 5,3

Peimbert M., Luridiana V., Peimbert A., 2007a, The Astrophysical Journal, 666, 636

Peimbert M., Luridiana V., Peimbert A., Carigi L., 2007b, On the primordial helium abundance and the deltay/deltao ratio. Tech. rep.

Peimbert M., Torres-Peimbert S., 1974, The Astrophysical Journal, 193, 327

Pequignot D., Petitjean P., Boisson C., 1991, Astronomy and Astrophysics, 251, 680

Pérez-Montero E., 2014, Monthly Notices of the Royal Astronomical Society, 441, 2663

Pérez-Montero E., Díaz A. I., 2003, Monthly Notices of the Royal Astronomical Society, 346, 105

Pérez-Montero E., Díaz A. I., 2005, 1063-1076

Pérez-Montero E., Kehrig C., Brinchmann J., Vílchez J. M., Kunth D., Durret F., 2013, Advances in Astronomy, 2013

Pilyugin L. S., Vílchez J. M., Thuan T. X., 2006, Monthly Notices of the Royal Astronomical Society, 370, 1928

Planck Collaboration et al., 2016, Astronomy and Astrophysics, 594, A13

Podobedova L. I., Kelleher D. E., Wiese W. L., 2009, Journal of Physical and Chemical Reference Data, 38, 171

Porter R. L., Ferland G. J., Storey P. J., Detisch M. J., 2012, Monthly Notices of the Royal Astronomical Society, 425, L28

Porter R. L., Ferland G. J., Storey P. J., Detisch M. J., 2013, Monthly Notices of the Royal Astronomical Society: Letters, 433, L89

Pradhan A. K., Montenegro M., Nahar S. N., Eissner W., 2006,
Monthly Notices of the Royal Astronomical Society: Letters, 366, L6

Ramsbottom C. A., Bell K. L., 1997, Atomic Data and Nuclear Data Tables, 66, 65

Rosales-Ortega F. F., 2006, Master's degree thesis, Instituto Nacional de Astrofísica en Óptica y Electrónica, Tonantzintla, Puebla, México

Sánchez Almeida J., Pérez-Montero E., Morales-Luis A. B., Muñoz-Tuñón C., García-Benito R., Nuza S. E., Kitaura F. S., 2016, The Astrophysical Journal, 819, 110

Songaila A., Cowie L., Hogan C., Rugers M., 1994, Nature, 368, 599

Storey P. J., Hummer D. G., 1995, Monthly Notices of the Royal Astronomical Society, 272, 41

Storey P. J., Zeippen C. J., 2000, Monthly Notices of the Royal Astronomical Society, 312, 813

Tayal S. S., 2000, The Astrophysical Journal, 530, 1091

Tayal S. S., 2007, The Astrophysical Journal Supplement Series, 171,331

Tayal S. S., 2011, The Astrophysical Journal Supplement Series, 195,12

Tayal S. S., Zatsarinny O., 2010, The Astrophysical Journal Supplement Series, 188, 32

Terlevich R., Terlevich E., Bosch G., Díaz A., Hägele G., Cardaci M., Firpo V., 2014, Monthly Notices of the Royal Astronomical Society, 445, 1449

Wiese W. L., Fuhr J. R., Deters T. M., 1996, Atomic transition probabilities of carbon, nitrogen, and oxygen : a critical data compilation

Zanstra H., 1931, Dominion Astrophys, 4, 209

Zeippen C. J., 1982, Monthly Notices of the Royal Astronomical Society, 198, 111

Zhang Y., Liu X., Wesson R., Storey P. J., Liu Y., Danziger I. J., 2004, Monthly Notices of the Royal Astronomical Society, 351, 935, arXiv: astro-ph/0403371 